\title{
A systematic review of studies measuring health-related quality of life of general injury populations: update 2010-2018
}

\author{
A. J. L. M. Geraerds ${ }^{1 *} \mathbb{D}$, Amy Richardson ${ }^{2 \dagger}$, Juanita Haagsma', Sarah Derrett ${ }^{2}$ and Suzanne Polinder ${ }^{1}$
}

\begin{abstract}
Background: Studies examining the impact of injury on health-related quality of life (HRQL) over time are necessary to understand the short- and long-term consequences of injury for population health. The aim of this systematic review was to provide an evidence update on studies that have measured HRQL over time in general injury populations using a generic (general) health state measure.
\end{abstract}

Methods: Studies conducted between 2010 and 2018 that assessed HRQL at more than one time point among general injury populations were eligible for inclusion. Two reviewers independently extracted information from each study on design, HRQL measure used, method of HRQL measure administration, timing of assessment(s), predictive variables, ability to detect change, and findings. Quality appraisals of each study were also completed by two reviewers using items from the RTI Item Bank on Risk of Bias and Precision of Observational Studies and the Guidelines for the Conduction of Follow-up Studies Measuring Injury-Related Disability.

Results: Twenty-nine studies (44 articles) that met the inclusion criteria were identified. HRQL was measured using 14 different generic measures; the SF-36, SF-12, and EQ-5D were used most frequently. A varying number of follow-up assessments were undertaken, ranging from one to five. Follow-up often occurred 12 months post-injury. Fewer studies $(n=11)$ examined outcomes two or more years post-injury, and only one to 10 years post-injury. While most studies documented improvements in HRQL over time since the injury event, study populations had not returned to pre-injury status or reached general population norm HRQL values at post-injury follow-ups.

Conclusions: Since 2010 there has been a substantial increase in the number of studies evaluating the HRQL of general injury populations. However, significant variability in study design continues to impede quantification of the impact of injury on population health over time. Variation between studies is particularly evident with respect to timing and number of follow-up assessments, and selection of instruments to evaluate HRQL.

Keywords: Health-related quality of life, Injuries, Systematic review

\footnotetext{
* Correspondence: a.geraerds@erasmusmc.nl

${ }^{\dagger}$ A. J. L. M. Geraerds and Amy Richardson contributed equally to this work.

'Department of Public Health, Erasmus MC, University Medical Center

Rotterdam, P.O. Box 2040, 3000 Rotterdam, CA, The Netherlands

Full list of author information is available at the end of the article
}

(C) The Author(s). 2020 Open Access This article is licensed under a Creative Commons Attribution 4.0 International License, which permits use, sharing, adaptation, distribution and reproduction in any medium or format, as long as you give appropriate credit to the original author(s) and the source, provide a link to the Creative Commons licence, and indicate if changes were made. The images or other third party material in this article are included in the article's Creative Commons licence, unless indicated otherwise in a credit line to the material. If material is not included in the article's Creative Commons licence and your intended use is not permitted by statutory regulation or exceeds the permitted use, you will need to obtain permission directly from the copyright holder. To view a copy of this licence, visit http://creativecommons.org/licenses/by/4.0/ The Creative Commons Public Domain Dedication waiver (http://creativecommons.org/publicdomain/zero/1.0/) applies to the data made available in this article, unless otherwise stated in a credit line to the data. 


\section{Background}

Worldwide, the global burden of disability continues to increase as a consequence of population growth, reductions in mortality due to improvements in healthcare, and the ageing of populations [1]. This presents a significant challenge for health systems which face growing demand for services designed to reduce the impact of disability on quality of life [2]. Injury has been identified as a key contributor to the global disability burden, particularly in high and middle-income countries [1]. Despite a notable decline in deaths from injury over time, non-fatal injuries remain a leading cause of hospitalisation [3]. The age-adjusted annualised rate of injuries requiring some form of medical treatment was approximately 126 per 1000 members of the United States (US) population in 2014 [4]. Current information regarding the impact of injury on subsequent disability is essential to plan for the effective allocation of available resources within health systems in order to promote optimum recovery from injury. This information can also be disseminated to patients to ensure they have accurate expectations for their recovery, and may be useful in the development of targeted interventions designed to minimise disability after injury.

While some information is available on the incidence of both fatal and nonfatal injuries, these data do not adequately depict the long-term consequences for injured individuals [3]. As a result, measures of health-related quality of life (HRQL), often assessing functional status (an important component of disability) [5] are increasingly utilised to quantify the effect of injury on population health [6]. HRQL measures, including generic and disease-specific measures, aim to provide a comprehensive estimation of health, and are often self-reported [7]. When examining outcomes following injury it is useful to use generic HRQL measures as these enable comparison of outcomes and recovery patterns within and between different injury populations [8]. Such measures also allow for comparisons between injured individuals and members of the general population, and with people with other health conditions [9]. This information can be used to inform approaches to rehabilitation and effective community reintegration.

Most generic HRQL measures are comprised of items that aim to measure health in relation to a broad range of dimensions, such as physical health, psychological health, mobility, social relationships, and environmental health [10]. There are different approaches to the reporting of findings obtained using these measures. Some studies report the proportion of individuals experiencing difficulties with respect to particular HRQL dimensions, while others report summary scores for each dimension (e.g. means and standard deviations/confidence intervals), and/or a global HRQL score based on the sum of all items within the measure. Some measures derive utility scores (weights) which are often determined by asking members of the general population to provide their 'preferences' for certain health states. Utility scores are commonly used in economic evaluations, incorporating the impact of injury on both quantity and quality of life [11]. Although there are various approaches to reporting findings from measures of HRQL, each approach can be used to understand patterns of HRQL over time for people with a broad range of injuries, highlighting potential pathways to recovery.

An earlier systematic review was conducted to examine studies that had measured HRQL using a generic instrument among general injury populations, in order to summarise existing knowledge in this area [12]. The review included studies conducted during 1995-2009 and found a lack of consensus on preferred HRQL instruments and study designs for the measurement of injuryrelated outcomes [12]. A total of 24 different generic HRQL and functional status measures were identified in the 41 studies meeting inclusion criteria. The most frequently used measures included the Medical Outcome Study Short Form-36 items (SF-36), the Functional Independence Measure (FIM), the Glasgow Outcome Scale (GOS), and the EQ-5D-3 L. These measures were found to be administered at a range of different times points post-injury, with follow-up most commonly occurring at 6, 12 and 24 months. Twelve studies reported HRQL utility scores. Overall, studies found that while significant recovery occurred in the first year post-injury, deficits from full recovery continued up to 2 years postinjury (when compared with population norms or preinjury health status) [12]. This was observed among populations with a broad range of injury severities, as well as severely injured populations.

Given the increasingly recognised importance of documenting the HRQL outcomes experienced by specific subpopulations, including individuals with injury [13], it is expected that many additional studies will have used generic health state measures among general injury populations since 2009 [14, 15]. However, it is unclear exactly how many studies have been conducted, how studies reported HRQL findings, and whether there has been greater consistency in study designs (including use of HRQL instruments, study populations, and assessment time points). It is possible that greater consistency in study designs may have been facilitated by the publication of the European Consumer Safety Association guidelines for undertaking follow-up studies measuring injury-related disability in 2007 [16]. These guidelines recommend the use of both the EQ-5D and Health Utilities Mark III (HUI) in all studies examining injuryrelated disability, with assessments at 1, 2, 4 and 12 months post-injury in addition to a pre-injury 
assessment. The earlier systematic review concluded that the guidelines were not being followed; yet this may have been because included studies had already finalised their protocol and/or data collection prior to the publication of the guidelines.

In order to gain contemporary information on injury outcomes and to investigate whether there has been an increase in the consistency of study designs since 2009 we conducted an updated systematic review of studies measuring HRQL with a generic instrument in general injury populations. Increased consistency in study designs would allow for improved comparisons between studies and increased precision in estimates of the burden of injury over time. As in the earlier review, we aimed to identify: i) which generic HRQL measures were used; ii) what methods were used to administer the measures; iii) the time points at which HRQL was measured; iv) how HRQL findings were reported; and v) whether changes over time, and predictors of, HRQL were assessed. We also explored whether studies eligible for inclusion used HRQL measures with properties that meet widely accepted recommendations in the field (with respect to internal consistency, reliability, measurement error, content validity, construct validity, criterion validity, responsiveness, and interpretability) [17]. Studies using appropriate measures and consistent designs are essential to ensure that accurate information on the burden of injury is available, allowing for the effective targeting of resources to maintain HRQL after injury.

\section{Methods}

\section{Data sources and strategy}

A new search of empirical studies on the HRQL of general injury populations was conducted. The search strategy that was developed for the systematic review of Polinder et al. [12] was updated in collaboration with a librarian specialising in literature searches. In order to match the database specific indexing terms, the search strategy was adjusted for the different electronic databases: Embase, PubMed (Medline Ovid), Web of Science and PsycINFO. The terms used in the search strategy were: 'quality of life' and 'health related quality of life', 'functional status assessment', 'injury' and 'trauma', and 'cohort analysis' (complete search strategy in Appendix 1). Articles were included in the search if the period of publication was between 2010 and 2018, and if they were peer-reviewed. The reference lists of the included articles were also screened, in order to detect additional articles that were relevant, and to identify important key terms. Details of the systematic review process were successfully registered and published within the PROSPERO database (registration number CRD42019120207).

\section{Selection criteria}

To be included in this review, studies had to use a generic HRQL or disability measure at more than one time point in a population of injury/trauma patients. While HRQL and disability are unique constructs, the World Health Organization International Classification of Functioning, Disability and Health (ICF) acknowledges the relationship between disability and HRQL, particularly with respect to participation in activities of daily living [5]. For the purpose of this review, the World Health Organization (WHO) definition of disability is used. The WHO defines disability as an umbrella term reflecting impairments, activity limitations, and participation restrictions [18]. The concept of HRQL is more specific, reflecting an individual's or population's perceptions of health (mental and physical) and functional status [19]. Several measures of disability, such as the World Health Organization Disability Assessment Schedule (WHODAS) based on the ICF, can be used to evaluate not only disability but also HRQL [20].

Additional inclusion criteria were publication in English and in a peer-reviewed journal between 2010 and 2018. Studies that focused on only one specific injury population, such as traumatic brain injury patients, were excluded as only studies with a general injury population were the focus of this review. Furthermore, studies measuring HRQL in people other than individuals with injury were excluded, as were studies employing nongeneric HRQL instruments, and review and pilot studies. There was no restriction on age or injury severity. Therefore, studies focusing on a specific age group or specific injury severity, but not focusing on a specific injury, were included.

\section{Data extraction and quality assessment}

After completion of the database searches, relevant articles were selected in three steps. First, the titles of the articles were screened, next, the abstracts of the articles selected in step one were screened, and finally, the entire articles selected in step two were read. By screening the titles, abstracts and articles, it was determined whether an article should be included or not according to the selection criteria. The screening procedure was conducted by two researchers independently ( $\mathrm{AG}$ and $\mathrm{AR}$ ). In cases of disagreement between the two researchers, a third researcher $(\mathrm{JH})$ was consulted. This researcher also checked a sample of abstracts $(n=50)$ in order to quality assure the process. The full articles that were eligible for inclusion were then analysed by two reviewers (AG and AR), using a modified version of the data extraction form developed for the original review by Polinder et al. [12]

The methodological quality of each study was independently assessed by two researchers (AG and AR) using three items from the RTI Item Bank on Risk of 
Bias and Precision of Observational Studies [21]. This item bank consists of 29 items designed to evaluate the quality of observational studies of interventions or exposures. It is recommended to select items that can evaluate the most critical threats to validity associated with the studies under investigation. For this review, items 16,17 , and 18 were selected for use; each of these items address potential bias associated with follow-up assessments in longitudinal studies. In addition, alignment of studies with the Guidelines for the Conduction of Follow-up Studies Measuring Injury-Related Disability was analyzed [16].

The results of all studies were tabulated in order to identify the different measures used, the methods of reporting HRQL information (e.g. summary scores), and whether any changes in HRQL over time were observed. For studies presenting HRQL summary scores, the scores could range from either 0 to 1 or 0 to 100 depending on the measurement instrument used. Two examples of generic HRQL instruments that can be used to derive a summary score are the EQ-5D and the SF36 . With respect to disability, an example of an instrument that can be used to derive a summary score is the WHODAS II [22]. For all instruments examined, lower scores were representative of worse health.

\section{Results}

\section{Literature search}

The search strategy in the specified databases provided a total of 8152 unique potentially relevant articles (see Fig. 1). One additional article that did not turn up in our search was extracted from the reference list of an included study, and added to the relevant titles. In the first selection round, based on scanning the titles, 7386 articles were excluded. The main reasons for exclusion were that studies were not about injury or were about a specific injury type, rather than injury in general. The abstracts of the remaining 766 articles were read in the next selection round, resulting in the exclusion of 668 more articles due to a lack of HRQL measurement. The full texts of the remaining 98 articles were read, and led to the final inclusion of 44 articles. These articles represent 29 unique studies. The main reason for final exclusion of 54 articles was a lack of a sufficient HRQL measurement or the lack of multiple HRQL measurements.

\section{Study characteristics}

Study characteristics are presented in Table 1. Out of the 44 articles that were included in our systematic review, most $(n=12)$ reported findings from a single prospective cohort study conducted in New Zealand [14,

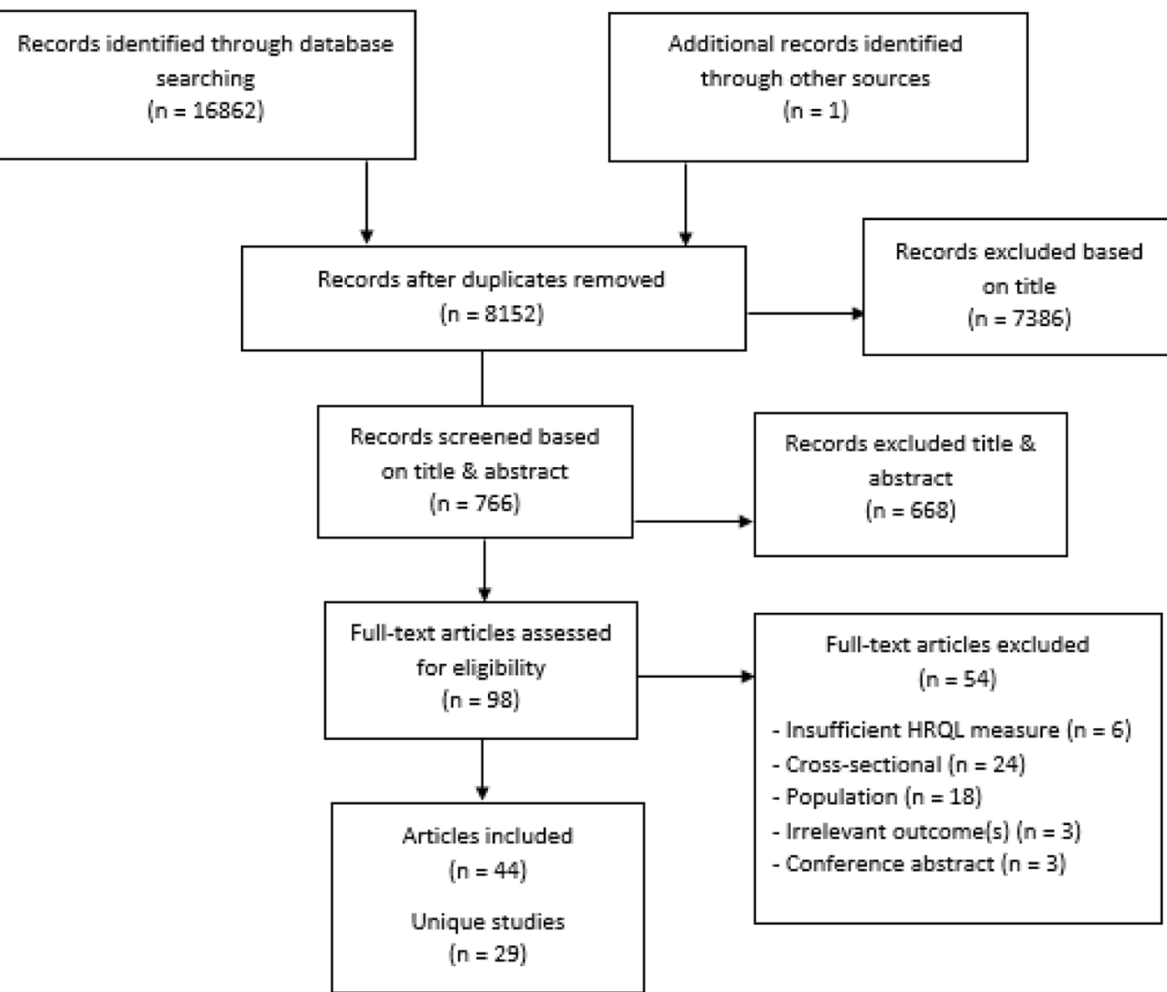

Fig. 1 PRISMA Flow Diagram 
Table 1 Study characteristics of included articles measuring HRQL in general injury populations

\begin{tabular}{|c|c|c|c|c|c|}
\hline $\begin{array}{l}\text { Author, year, } \\
\text { country }\end{array}$ & Study population and design & HRQL instrument & $\begin{array}{l}\text { Follow up time } \\
\text { points }\end{array}$ & Predictors of HRQL/Disability & Outcomes \\
\hline $\begin{array}{l}\text { Abedzadeh- } \\
\text { Kalahroudi, } 2015 \\
\text { [23], Iran }\end{array}$ & $\begin{array}{l}\text { Hospitalised trauma patients } \\
(15-65 y)(N=400) ; \text { Hospital; } \\
\text { Prospective cohort study }\end{array}$ & WHODAS II & $\begin{array}{l}1 \text { month } \\
3 \text { months }\end{array}$ & $\begin{array}{l}\text { Predictors disability: age, } \\
\text { length of hospital stay, injury } \\
\text { to extremities }\end{array}$ & $\begin{array}{l}\text { Disability: } \\
-1 \text { month mean: } 30.3 \\
\text { (9.2) } \\
\text { - } 3 \text { months mean: } 18.8 \\
\quad \text { (8.3) } \\
\text { - Activity limitation: } 11.3 \\
\quad \text { (15.8) } \\
\text { - Participation: } 16.9 \\
\text { (20.2) }\end{array}$ \\
\hline
\end{tabular}

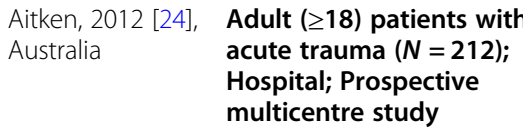

SF-36

$\begin{array}{ll} & \begin{array}{l}\text { Hospital; Prospective } \\ \text { multicentre study }\end{array} \\ & \\ \text { Aitken, 2014 [25], } & \text { Trauma intensive care } \\ \text { Australia } & \begin{array}{l}\text { patients (adults) from one } \\ \text { tertiary referral hospital } \\ \text { admitted for acute injury } \\ (N=123) ; \text { Prospective cohort } \\ \text { study }\end{array}\end{array}$

\section{SF-36}

Hospital discharge (92\%) 3 months $(60 \%)$ 6 months (59\%)

Psychological status: Kessler Psychological Distress Scale (K10) and the PTSD Civilian Checklist

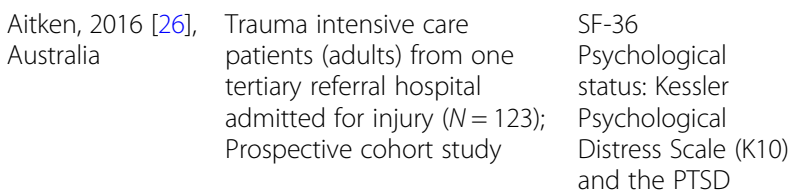
patients (adults) from one tertiary referral hospital admitted for injury $(N=123)$; Prospective cohort study

\section{SF-36}

Psychological

status: Kessler

Psychological

Distress Scale (K10)

and the PTSD

Davie, 2018 [27], New Zealand

Individuals (18-64y) from ACC entitlement claims register $(N=2856)$;

Prospective cohort study
Civilian Checklist
1 month (76\%)

6 months $(72 \%)$

12 months (68\%)

24 months (56\%)

Non-modifiable factors linked with physical function:

Optimistic perception of illness, greater self-efficacy, hospital length of stay, injury insurance

\section{WHODAS II}

3 months

12 months

24 months $(65 \%$ with complete data)

Comorbidity
PCS: age, body region $y$, perceived MCS: age, gender, perceived ability to control environment predicted outcome

Not identified
Slight improvement in HRQL from 3 to 6 months after hospital discharge, but not back at pre-injury level

HRQL outcome:

- 1 months: PCS: 32.7

(10.4); MCS: 40.6 (15.7)

- 6 months: PCS: 40.9

(13.2); MCS: 42.6 (14.0)

Scores significantly

below Australian norms both 1 and 6 months

post-discharge

HRQL outcome:

- 1 month: PCS: 32.7

(10.4); MCS: 40.6 (15.7)

- 6 months: PCS: 40.9

(13.2); MCS: 42.6 (14.0)

- 12 months: PCS: 42.8

(11.7); MCS: 42.4 (13.8)

- 24 months: PCS: 43.7

(12.3); MCS: 44.6 (12.5)

Averages remained

below Australian norms

at 24 months

Percentage disabled:

-3 months: No

comorbidities: $37.2 \%$

1 comorbidity: $39.8 \%$

Multimorbidity: $\mathbf{5 1 . 9 \%}$

- 12 months: No

comorbidities: $10.6 \%$

1 comorbidity: $11.4 \%$

Multimorbidity: $27.1 \%$

- 24 months: No

comorbidities: $\mathbf{8 . 9 \%}$

1 comorbidities:

$10.8 \%$

Multimorbidity: $\mathbf{2 4 . 6 \%}$

EQ-5D +

cognition;

WHODAS II 12-

item

Prospective cohort study

Derrett, 2012 [28], Individuals (18-64y) from

WHODAS II
3 months (96\%) (informed on pre-injury status and post injury status in one interview)

3 months (59\%) Not identified (preliminary analysis only)

Associated with disability: disability experienced higher injury severity (NISS > 3), female, $\geq 2$ chronic conditions before injury, perceiving a threat of disability, lower extremity fracture
Worse HRQL and increased disability compared to preinjury status

Non-hospitalised: by $39 \% 3$ months after injury Hospitalised: Phase disability more prevalent 
Table 1 Study characteristics of included articles measuring HRQL in general injury populations (Continued)

\begin{tabular}{|c|c|c|c|c|}
\hline $\begin{array}{l}\text { Author, year, } \\
\text { country }\end{array}$ & Study population and design & HRQL instrument & $\begin{array}{l}\text { Follow up time } \\
\text { points }\end{array}$ & Predictors of HRQL/Disability \\
\hline $\begin{array}{l}\text { Derrett, } 2013 \text { [29], } \\
\text { New Zealand }\end{array}$ & $\begin{array}{l}\text { Individuals }(18-64 y) \text { from } \\
\text { ACC entitlement claims } \\
\text { register }(N=2856) ; \\
\text { Prospective cohort study }\end{array}$ & WHODAS II & 24 months (76\%) & $\begin{array}{l}\text { Post-injury disability: } \\
\text { - Hospitalised: WHODAS } \geq \\
10, \geq 2 \text { chronic conditions } \\
\text { pre-injury, not being opti- } \\
\text { mistic pre-injury, BMI } \geq 30 \text {, } \\
\text { smoking, perceived threat } \\
\text { of long term disability, } \\
\text { trouble accessing health } \\
\text { care, head/neck superficial } \\
\text { injury, lower extremity } \\
\text { open wound } \\
\text { - Non-hospitalised: WHO- } \\
\text { DAS } \geq 10, \geq 2 \text { chronic con- } \\
\text { ditions pre-injury, } \\
\text { depressive type episode } \\
\text { pre-injury, BMI } \geq 30, \text { smok- } \\
\text { ing, intentional injury, } \\
\text { trouble accessing health } \\
\text { care, intracranial injury, } \\
\text { spine sprain/dislocation }\end{array}$ \\
\hline
\end{tabular}

\author{
Harcombe, 2015 Individuals (18-64y) from \\ [30], New \\ Zealand \\ ACC entitlement claims \\ register $(N=2856)$;
}

Prospective cohort study

Langley, 2013

[31], New

Zealand
Maclennan, 2013 Individuals of Māori

[32], New

Zealand

ethnicity from ACC
Individuals (18-64y) from ACC entitlement claims register $(N=2856)$;

Prospective cohort study
EQ-5D

3 months

12 months

24 months (25-

$28 \%$ missing at

least 1 response)

EQ-5D +

cognition

3 months

12 months (80\%)

EQ-5D +

cognition;

3 months (59\%)

entitlement claims register

(18-64y) $(N=566) ;$

Prospective cohort study

WHODAS II 12

item
Maclennan, 2014 Individuals (18-64y) from [33], New

Zealand
ACC entitlement claims

register $(N=2856)$;

Prospective cohort study
WHODAS II \& EQ- 3 months $5 \mathrm{D}+$ cognition 12 months $(80 \%)$
Not identified

Not identified

Preinjury EQ-5D status, fe-

male, age 45-64, inadequate household income, preinjury disability, 2 or more prior chronic illnesses, smoking regularly, dislocation/sprains to spine or upper extremities, having relatively severe injury

Not identified

HRQL:

- Walking difficulties:

+/- half cohort

- Pain/discomfort: 2/3

of cohort

- Psychological distress: $>1 / 2$ cohort

- Disability: $49 \%$

- Satisfied with life: majority

- Consider themselves in good/excellent health: majority

Pre-injury:

- Non-Māori: > 90\% good health

- Māori: > 90\% good health

12 months:

- Non-Māori: prob-

lems increased 4$40 \%$

- Māori: problems

increased $5-45 \%$ 
Table 1 Study characteristics of included articles measuring HRQL in general injury populations (Continued)

\begin{tabular}{|c|c|c|c|c|c|}
\hline $\begin{array}{l}\text { Author, year, } \\
\text { country }\end{array}$ & Study population and design & HRQL instrument & $\begin{array}{l}\text { Follow up time } \\
\text { points }\end{array}$ & Predictors of HRQL/Disability & Outcomes \\
\hline $\begin{array}{l}\text { [34], New } \\
\text { Zealand }\end{array}$ & $\begin{array}{l}\text { ACC entitlement claims } \\
\text { register }(N=2856) ; \\
\text { Prospective cohort study }\end{array}$ & WHODAS II & & $\begin{array}{l}\text { with disability \& HRQL: } \\
\text { Pacific people }\end{array}$ & $\begin{array}{l}\text { likely to have: } \\
\text { - Disability: no/lesser } \\
\text { problems } \\
\text { - Self-care: no } \\
\text { problems } \\
\text { - Anxiety/depression: } \\
\text { no problems }\end{array}$ \\
\hline $\begin{array}{l}\text { Wilson, } 2013 \text { [35], } \\
\text { New Zealand }\end{array}$ & $\begin{array}{l}\text { Individuals (18-64y) from } \\
\text { ACC entitlement claims } \\
\text { register }(N=2856) ; \\
\text { Prospective cohort study }\end{array}$ & $\begin{array}{l}\mathrm{EQ}-5 \mathrm{D}+ \\
\text { cognition }\end{array}$ & 12 months (78\%) & $\begin{array}{l}\text { Sex, injury severity, } \\
\text { hospitalisation status }\end{array}$ & $\begin{array}{l}\text { Mean QALYs lost first } \\
\text { year after injury: } \\
\text { - Male: } 0.21 \text { QALY } \\
\text { - Female: } 0.24 \text { QALY } \\
\text { - Hospitalised: } 0.25 \\
\text { QALY } \\
\text { - Non-hospitalised: } \\
0.21 \text { QALY }\end{array}$ \\
\hline $\begin{array}{l}\text { Wyeth, } 2017 \text { [36], } \\
\text { New Zealand }\end{array}$ & $\begin{array}{l}\text { Individuals (18-64y) from } \\
\text { ACC entitlement claims } \\
\text { register }(N=2856) ; \\
\text { Prospective cohort study }\end{array}$ & WHODAS II & $\begin{array}{l}3 \text { months } \\
24 \text { months (66\%) }\end{array}$ & $\begin{array}{l}\text { Disability at } 24 \text { months: } \geq 2 \\
\text { chronic conditions pre- } \\
\text { injury, trouble accessing } \\
\text { healthcare services after in- } \\
\text { jury; hospitalisation for in- } \\
\text { jury, inadequate pre-injury } \\
\text { household income }\end{array}$ & $\begin{array}{l}\text { Percent disability: } \\
\text { - Pre-injury: } 9 \% \\
\text { - } 24 \text { months: } 19 \% \\
\text { - Age } 30-49: 23 \% \\
\text { (highest proportion) }\end{array}$ \\
\hline $\begin{array}{l}\text { Wyeth, } 2018 \text { [37], } \\
\text { New Zealand }\end{array}$ & $\begin{array}{l}\text { Individuals }(18-64 y) \text { from } \\
\text { ACC entitlement claims } \\
\text { register }(N=2856) \text {; } \\
\text { Prospective cohort study }\end{array}$ & WHODAS & $\begin{array}{l}24 \text { months (80\% } \\
\text { non-Māori; } 66 \% \\
\text { Māori) }\end{array}$ & $\begin{array}{l}\text { Māori: not working for pay } \\
\text { before injury, experiencing } \\
\text { disability before injury, } \\
\text { trouble accessing } \\
\text { healthcare services for } \\
\text { injury } \\
\text { Non-Māori: inadequate } \\
\text { household income prior to } \\
\text { injury, less than secondary } \\
\text { school qualifications, not } \\
\text { working for pay, disability } \\
\text { prior to injury, } \geq 2 \text { chronic } \\
\text { conditions, BMI } \geq 30\end{array}$ & $\begin{array}{l}\text { RR of disability } 24 \\
\text { months after injury: } \\
\text { Māori: } \\
\text { - Hospitalised, non- } \\
\text { working: } 2.7 \text { (1.4, } \\
4.9) \\
\text { - Pre-injury disabled: } \\
3.1(1.6,5.8) \\
\text { - Difficulties accessing } \\
\text { health care: } 2.6(1.3 \text {, } \\
5.2) \\
\text { Non-Māori: } \\
\text { - Hospitalised, } \\
\text { inadequate } \\
\text { household income: } \\
2.4(1.4,4.1) \\
\text { - Less than secondary } \\
\text { school qualification: } \\
2.0(1.1,3.8) \\
\text { - Not working for pay } \\
\text { before injury: } 2.8 \\
\text { (1.5, } 5.1) \\
\text { - Disability before } \\
\text { injury: } 3.0(1.7,5.2) \\
\text { - } \geq 2 \text { chronic } \\
\text { conditions: } 3.5(2.0, \\
6.4) \\
\text { - BMI } \geq 30: 2.4 \text { (1.3, } \\
4.4 .)\end{array}$ \\
\hline $\begin{array}{l}\text { Dhungel, } 2015 \\
\text { [38], US }\end{array}$ & $\begin{array}{l}\text { Adult }(18+) \text { trauma } \\
\text { population divided in groups } \\
\text { of normal weight, } \\
\text { overweight, obese and } \\
\text { morbidly obese }(N=235) \text {; } \\
\text { Trauma centre; Prospective } \\
\text { cohort study }\end{array}$ & FIM & $\begin{array}{l}\text { Admission Hospital } \\
\text { discharge } \\
6 \text { months (79\%) }\end{array}$ & Not defined & $\begin{array}{l}\text { Functional Status: } \\
\text { - Admission: Non- } \\
\text { obese: } 38.2 \text { (13.9) } \\
\text { Overweight: } 40.0 \text { (11.1) } \\
\text { Obese: } 38.3 \text { (15.1) } \\
\text { Morbidly obese: } 41.6 \\
\text { (13.9) } \\
\text { - Discharge: Non-obese: } \\
62.4 \text { (7.9) } \\
\text { Overweight: } 60.0 \text { (8.4) } \\
\text { Obese: } 56.7 \text { (13.0) } \\
\text { Morbidly obese: } 58.7 \\
\text { (9.3) } \\
\text { - Follow-up: Non-obese: }\end{array}$ \\
\hline
\end{tabular}


Table 1 Study characteristics of included articles measuring HRQL in general injury populations (Continued)

\begin{tabular}{|c|c|c|c|c|c|}
\hline $\begin{array}{l}\text { Author, year, } \\
\text { country }\end{array}$ & Study population and design & HRQL instrument & $\begin{array}{l}\text { Follow up time } \\
\text { points }\end{array}$ & Predictors of HRQL/Disability & Outcomes \\
\hline & & & & & $\begin{array}{l}71.1(2.1) \\
\text { Overweight: } 70.6(3.4) \\
\text { Obese: } 70.3 \text { (3.8) } \\
\text { Morbidly obese: } 69.8 \\
(5.4)\end{array}$ \\
\hline $\begin{array}{l}\text { Dinh, } 2016[39], \\
\text { Australia }\end{array}$ & $\begin{array}{l}\text { Adult }(\geq 16) \text { trauma } \\
\text { patients }(N=349) ; \text { Major } \\
\text { trauma centre; Prospective } \\
\text { cohort study }\end{array}$ & EQ-5D and SF-12 & $\begin{array}{l}\text { Baseline } \\
3 \text { months } \\
6 \text { months (51\%) }\end{array}$ & $\begin{array}{l}\text { Physical health: lower limb } \\
\text { injuries; Mental health: } \\
\text { mechanism of injury, past } \\
\text { mental health; RTW: } \\
\text { increasing ISS, upper limb } \\
\text { injuries }\end{array}$ & $\begin{array}{l}\text { HRQL: No significant } \\
\text { change in PCS and } \\
\text { MCS between } 3 \text { and } \\
6 \text { months }\end{array}$ \\
\hline $\begin{array}{l}\text { Gabbe, } 2013[40], \\
\text { Australia }\end{array}$ & $\begin{array}{l}\text { Adult major trauma patients } \\
(N=662) \text {; Level } 1 \text { trauma } \\
\text { centre; Prospective cohort } \\
\text { study }\end{array}$ & $\begin{array}{l}\text { SF-12 } \\
\text { GOSE }\end{array}$ & $\begin{array}{l}6 \text { months } \\
12 \text { months } \\
18 \text { months } \\
24 \text { months ( } 93 \% \\
\text { followed up for at } \\
\text { least } 1 \text { time point) }\end{array}$ & Not defined & $\begin{array}{l}\text { - 6-12 months: Func- } \\
\text { tional recovery, RTW, } \\
\text { physical health } \\
\text { improved } \\
\text { - >12 months: little } \\
\text { change } \\
\text { - <18 months: mental } \\
\text { health score } \\
\text { decreased } \\
\text { - } 18-24 \text { months: mental } \\
\text { health score improved }\end{array}$ \\
\hline $\begin{array}{l}\text { Gabbe, } 2016[41], \\
\text { Australia }\end{array}$ & $\begin{array}{l}\text { Adult major trauma } \\
\text { survivors }(N=8844) ; \\
\text { Victorian State Trauma } \\
\text { Registry (VSTR); } \\
\text { Prospective cohort study }\end{array}$ & $\begin{array}{l}\text { GOS } \\
\text { GOSE }\end{array}$ & $\begin{array}{l}6 \text { months } \\
12 \text { months } \\
24 \text { months ( } 74 \% \\
\text { for all follow-up } \\
\text { points) }\end{array}$ & $\begin{array}{l}\text { Female, older patients, pre- } \\
\text { existing conditions, spinal } \\
\text { cord injured and multi- } \\
\text { trauma patients involving } \\
\text { head injury, intentional/low- } \\
\text { fall events, compensable } \\
\text { patients, greater socioeco- } \\
\text { nomic disadvantage, pre- } \\
\text { existing drug/alcohol/men- } \\
\text { tal health conditions }\end{array}$ & $\begin{array}{l}\text { Good recovery: } \\
\text { - } 6 \text { months: Male: } \\
33.2 \% \text {; Female: } \\
27.2 \% \\
\text { - } 12 \text { months: Male: } \\
37.3 \% \text {; Female: } \\
28.8 \% \\
\text { - 24 months: Male: } \\
39.7 \% \text {; Female: } \\
31.1 \%\end{array}$ \\
\hline $\begin{array}{l}\text { Gabbe, } 2017[42], \\
\text { Australia }\end{array}$ & $\begin{array}{l}\text { Hospitalised adult major } \\
\text { trauma patients }(\text { ISS } \geq 12) \\
(N=2424) ; \text { Victorian State } \\
\text { Trauma Registry (VSTR); } \\
\text { Prospective cohort study }\end{array}$ & EQ-5D-3 L & $\begin{array}{l}6 \text { months }(84 \%) \\
12 \text { months }(85 \%) \\
24 \text { months }(84 \%) \\
36 \text { months }(74 \%)\end{array}$ & $\begin{array}{l}\text { Age, compensable status, } \\
\text { level of education, nature of } \\
\text { injuries, gender, preinjury } \\
\text { employment, level of } \\
\text { socioeconomic } \\
\text { disadvantage }\end{array}$ & $\begin{array}{l}\text { HRQL:- } 6 \text { months: } 0.67 \\
(0.31) \\
-12 \text { months: } 0.68 \\
(0.32) \\
-24 \text { months: } 0.71 \\
(0.31) \\
-36 \text { months: } 0.70 \\
(0.32)\end{array}$ \\
\hline $\begin{array}{l}\text { Gross, } 2011[43] \\
\text { Switzerland }\end{array}$ & $\begin{array}{l}\text { Patients treated primarily at a } \\
\text { university trauma centre after } \\
\text { blunt polytrauma }(N=178) ; \\
\text { University hospital ICU; } \\
\text { Prospective cohort study }\end{array}$ & $\begin{array}{l}\text { EQ-5D } \\
\text { SF-36 } \\
\text { MFA } \\
\text { TOP }\end{array}$ & 24 months (57\%) & $\begin{array}{l}\text { Long term pain associated } \\
\text { with HRQL-scores }\end{array}$ & $\begin{array}{l}\text { Mean (SD) HRQL: } \\
\text { EQ-5D pain: } \\
\text { - Pre-injury: } 1.1(0.4) \\
\text { - Post-injury: } 1.7(0.6) \\
\text { SF-36 pain: } \\
\text { - Pre-injury: } 94.3(14.1) \\
\text { - Post injury: } 65.0(29.5) \\
\text { MFA pain: } \\
\text { - Pre-injury: } 1.4(0.7) \\
\text { - Post-injury: } 2.4(1.2) \\
\text { TOP total pain: } \\
\text { - Pre-injury: } 96.2(7.7) \\
\text { - Post injury: } 72.0(29.7)\end{array}$ \\
\hline $\begin{array}{l}\text { Gross, } 2012[44], \\
\text { Switzerland }\end{array}$ & $\begin{array}{l}\text { Polytrauma patients defined } \\
\text { as trauma victims with ISS } \geq \\
16(N=170) \text {; University } \\
\text { hospital ICU; Prospective } \\
\text { cohort study }\end{array}$ & $\begin{array}{l}\text { EQ-5D } \\
\text { SF-36 }\end{array}$ & 2.5 years $(65 \%)$ & $\begin{array}{l}\text { Negative association with EQ- } \\
5 \mathrm{D} \text { and SF-36: Brain injury }\end{array}$ & $\begin{array}{l}\text { HRQL: } \\
\text { EQ-VAS: } \\
\text { - Pre-injury: Non-TBI: } \\
\text { 88.5 (17.6); TBI: } 91.4 \\
\text { (9.5) } \\
\text { - Post-injury: Non-TBI: } \\
\text { 69.9 (23.4); TBI: } 59.4 \\
\text { (25.0) } \\
\text { EQ-5D: } \\
\text { - Pre-injury: Non-TBI: } \\
\text { 94.5 (13.7); TBI: } 98.6\end{array}$ \\
\hline
\end{tabular}


Table 1 Study characteristics of included articles measuring HRQL in general injury populations (Continued)

\begin{tabular}{|c|c|c|c|c|c|}
\hline $\begin{array}{l}\text { Author, year, } \\
\text { country }\end{array}$ & Study population and design & HRQL instrument & $\begin{array}{l}\text { Follow up time } \\
\text { points }\end{array}$ & Predictors of HRQL/Disability & Outcomes \\
\hline & & & & & $\begin{array}{l}\text { (3.6) } \\
\text { - Post-injury: Non-TBI: } \\
\text { 76.4 (20.8); TBI: } 65.4 \\
\text { (27.7) } \\
\text { SF-36: } \\
\text { - Pre-injury: } \\
\text { PCS: non-TBI: } 56.0 \text { (6.9); } \\
\text { TBI: } 56.8 \text { (5.5) } \\
\text { MCS: non-TBI: } 50.8 \\
\text { (11.8); TBI: } 50.3 \text { (11.3) } \\
\text { - Post-injury: } \\
\text { PCS: non-TBI: } 45.3 \text { (10.6); } \\
\text { TBI: 44.0 (11.9) } \\
\text { MCS: non-TBI: } 48.1 \\
\text { (12.9); TBI: } 38.9 \text { (13.1) }\end{array}$ \\
\hline $\begin{array}{l}\text { Gross, } 2019 \text { [45], } \\
\text { Switzerland }\end{array}$ & $\begin{array}{l}\text { Major trauma patients (15- } \\
63 y) \text { (NISS } \geq 8)(N=1078) ; \\
\text { Teaching hospital; } \\
\text { Prospective cohort study }\end{array}$ & $\begin{array}{l}\text { SF-36, EQ-5D \& } \\
\text { GOS }\end{array}$ & $\begin{array}{l}1 \text { year } \\
2 \text { years } \\
(31.2 \% \text { year } 1 \& \\
2)\end{array}$ & $\begin{array}{l}\text { Associated with GOS } \\
\text { outcomes between 1-2y } \\
\text { after trauma: gender, age, } \\
\text { trauma, energy, length of } \\
\text { hospital stay }\end{array}$ & $\begin{array}{l}\text { HRQL: } \\
\text { EQ-5D: } \\
-1 \text { year: } \\
\text { Male: } 0.74 \text { (0.22) } \\
\text { Female: } 0.77 \text { (0.19) } \\
\text { - } 2 \text { years: } \\
\text { Male: } 0.74 \text { (0.22) } \\
\text { Female: } 0.80 \text { (0.15) } \\
\text { SF-36: } \\
\text { - } 1 \text { year: } \\
\text { Male: PCS: } 46.11 \\
\text { (9.78); MCS: } 49.25 \\
\text { (12.66) } \\
\text { Female: PCS: } 47.54 \\
\text { (9.24); MCS: } 47.92 \\
\text { (11.81) } \\
-2 \text { years: } \\
\text { Male: PCS: } 46.29 \\
\text { (9.97); MCS: } 50.14 \\
\text { (12.78) } \\
\text { Female: PCS: } 48 \\
\text { 8(8.18); MCS: } 49.61 \\
\text { (10.60) }\end{array}$ \\
\hline $\begin{array}{l}\text { Innocenti, } 2014 \\
\text { [46], Italy }\end{array}$ & $\begin{array}{l}\text { Adult }(\geq 18) \text { patients } \\
\text { admitted in ED-HDU for } \\
\text { trauma ( } N=418) \text {; Prospective } \\
\text { cohort study }\end{array}$ & SF-12 & 6 months (58\%) & Not defined & $\begin{array}{l}\text { Pre-injury: } \\
\text { - MCS: normal score: } \\
\text { 94\% } \\
\text { - PCS: normal score: } \\
\text { 96\% } \\
\text { After injury: } \\
\text { - MCS: normal score: } \\
\text { 70\% } \\
\text { - PCS: normal score: } \\
\text { 58\% }\end{array}$ \\
\hline $\begin{array}{l}\text { Innocenti, } 2015 \\
{[47] \text {, Italy }}\end{array}$ & $\begin{array}{l}\text { Mild to moderate trauma } \\
\text { patients admitted to ED } \\
\text { high dependency unit ( } N= \\
\text { 286); Prospective cohort } \\
\text { study }\end{array}$ & SF-12 & 6 months $(53 \%)$ & $\begin{array}{l}\text { Older age, female, pre- } \\
\text { existing medical conditions, } \\
\text { high Sequential Organ Fail- } \\
\text { ure Assessment score }\end{array}$ & $\begin{array}{l}\text { Pre-injury: } \\
\text { - PCS: } 53 \text { (7) } \\
\text { - MCS: } 55 \text { (7) } \\
6 \text { months: } \\
\text { - PCS: } 41 \text { (12) } \\
\text { - MCS: } 46 \text { (13) } \\
\text { Maintain normal } \\
\text { value after injury: } \\
\text { PCS: } 52 \% \\
\text { MCS: } 68 \%\end{array}$ \\
\hline $\begin{array}{l}\text { Jagnoor, } 2017 \\
\text { [48], India }\end{array}$ & $\begin{array}{l}\text { Children (2-16y) with } \\
\text { overnight admission to } \\
\text { hospital due to injury ( } N= \\
\text { 386); Hospital/secondary/ } \\
\text { tertiary care institution; } \\
\text { Prospective multicentre study }\end{array}$ & PedsQL & $\begin{array}{l}\text { Pre-injury (97\%) } \\
1 \text { month (73\%) } \\
2 \text { months } \\
4 \text { months } \\
12 \text { months ( } 77 \% \text { all } \\
\text { time points) }\end{array}$ & Not defined & $\begin{array}{l}\text { Mean score: } \\
\text { - Baseline: } \\
\text { Physical score: } 99.4 \text { (3.4) } \\
\text { Psychosocial score: } 99.4 \\
\text { (3.4) } \\
\text { - } 1 \text { month: } \\
\text { Physical: } 79.7\end{array}$ \\
\hline
\end{tabular}


Table 1 Study characteristics of included articles measuring HRQL in general injury populations (Continued)

\begin{tabular}{|c|c|c|c|c|c|}
\hline $\begin{array}{l}\text { Author, year, } \\
\text { country }\end{array}$ & Study population and design & HRQL instrument & $\begin{array}{l}\text { Follow up time } \\
\text { points }\end{array}$ & Predictors of HRQL/Disability & Outcomes \\
\hline & & & & & $\begin{array}{l}\text { Psychosocial: } 86.3 \\
\text { - } 2 \text { months: all scores } \\
\text { improved }\end{array}$ \\
\hline $\begin{array}{l}\text { Kendrick, } 2017 \\
\text { [49], UK }\end{array}$ & $\begin{array}{l}\text { Patients }(16-70 y) \text { with } \\
\text { unintentional injury that } \\
\text { required hospital } \\
\text { admission }(N=668) ; \\
\text { Hospital; Prospective } \\
\text { multicentre study }\end{array}$ & EQ-5D-3 L & $\begin{array}{l}1 \text { month }(77 \%) \\
2 \text { months }(72 \%) \\
4 \text { months }(68 \%) \\
12 \text { months }(63 \%)\end{array}$ & $\begin{array}{l}\text { Associated with clinically } \\
\text { important reductions in } \\
\text { HRQL between } 2 \& 12 \\
\text { months post-injury: Higher } \\
\text { depression and anxiety } \\
\text { scores }\end{array}$ & $\begin{array}{l}\text { HRQL: } \\
\text { - Pre-injury: } 0.92 \\
(0.18) \\
-1 \text { month: } 0.44(0.28) \\
-2 \text { months: } 0.57(0.27) \\
-4 \text { months: } 0.69(0.23) \\
-12 \text { months: } 0.78 \\
(0.21) \\
60 \% \text { respondents } 12 \\
\text { months after injury } \\
\text { lower HRQL than pre- } \\
\text { injury }\end{array}$ \\
\hline $\begin{array}{l}\text { Llaquet, } 2018 \\
\text { [50], Spain }\end{array}$ & $\begin{array}{l}\text { Injured adult }(\geq 16) \text { patients } \\
\text { admitted to intensive care } \\
\text { unit in Spanish level } 1 \\
\text { trauma centre }(N=304) \text {; } \\
\text { Prospective cohort study }\end{array}$ & EQ-5D-5 L & $\begin{array}{l}\text { Hospital discharge } \\
3 \text { months } \\
6 \text { months } \\
12 \text { months (66\%) }\end{array}$ & $\begin{array}{l}\text { Lower EQ-VAS: Age } \geq 55 \text {, fe- } \\
\text { male, unskilled employment }\end{array}$ & $\begin{array}{l}\text { HRQL: } \\
\text { EQ-VAS: } \\
\text { - Discharge: } 60 \\
\text { - } 3 \text { months: } 65 \\
\text { - } 6 \text { months: } 70 \\
\text { - } 12 \text { months: } 75\end{array}$ \\
\hline $\begin{array}{l}\text { Nguyen, } 2018 \\
\text { [51], Vietnam }\end{array}$ & $\begin{array}{l}\text { Adult injury patients } \\
\text { hospitalised for at least } 1 \\
\text { day }(N=892) ; \text { Hospital; } \\
\text { Prospective cohort study }\end{array}$ & HUI3 & $\begin{array}{l}1 \text { month }(86 \%) \\
2 \text { months }(86 \%) \\
4 \text { months }(85 \%) \\
12 \text { months }(82 \%)\end{array}$ & $\begin{array}{l}\text { Older age, more severe } \\
\text { injury, other illnesses }\end{array}$ & $\begin{array}{l}\text { HRQL: } \\
\text { - } 1 \text { month: Males: } \\
\text { 0.52; Female: } 0.28 \\
\text { - } 2 \text { months: Males: } \\
\text { 0.67; Females: } 0.47 \text { I } \\
\text {-4 months: Males: } \\
\text { 0.77; Females: } 0.57 \\
\text { - } 12 \text { months: Males: } \\
\text { 0.87; Females: } 0.71\end{array}$ \\
\hline $\begin{array}{l}\text { Orwelius, } 2012 \\
\text { [52], Sweden }\end{array}$ & $\begin{array}{l}\text { Adult patients with } \\
\text { emergency admission to ICU } \\
(N=146) \text {; ICU; Prospective } \\
\text { multicentre study }\end{array}$ & SF-36 & $\begin{array}{l}6 \text { months }(74 \%) \\
12 \text { months (58\%) } \\
24 \text { months (39\%) }\end{array}$ & $\begin{array}{l}\text { Associated with HRQL: Pre- } \\
\text { existing disease, Maximum } \\
\text { SOFA score, APACHE-II score, } \\
\text { marital status }\end{array}$ & $\begin{array}{l}\text { - 6-12 months: signifi- } \\
\text { cant improvements } \\
\text { for role limitations } \\
\text { caused by physical } \\
\text { problems; improve- } \\
\text { ment in bodily pain } \\
\text { - 12-24 months: further } \\
\text { improvements }\end{array}$ \\
\hline $\begin{array}{l}\text { Pieper, } 2015[53] \\
\text { US }\end{array}$ & $\begin{array}{l}\text { Children 8-17 with mild } \\
\text { (brain) injury or no injury } \\
(N=120) \text {; Paediatric } \\
\text { emergency department; } \\
\text { Prospective cohort study }\end{array}$ & PedsQL & $\begin{array}{l}\text { Baseline } \\
\text { (preinjury) } \\
1 \text { month } \\
3 \text { months } \\
6 \text { months } \\
12 \text { months (86\%) }\end{array}$ & Not defined & $\begin{array}{l}\text { Total generic health: } \\
\text { - Baseline: Child: } 83.5 \\
\text { Parent: } 86.9 \\
\text { - } 1 \text { month: Child: } 83.1 \\
\text { Parent: } 84.2 \\
\text { - } 3 \text { months: Child: } 86.1 \\
\text { Parent: } 85.6 \\
\text { - } 6 \text { months: Child: } 87.4 \\
\text { Parent: } 85.7 \\
\text { - } 12 \text { months: Child: } \\
\text { 88.6 Parent: } 87.0\end{array}$ \\
\hline $\begin{array}{l}\text { Rainer, } 2014 \text { [54], } \\
\text { Hong Kong/ } \\
\text { Australia }\end{array}$ & $\begin{array}{l}\text { Adult }(\geq 18) \text { Major trauma } \\
\text { patients (ISS } \geq 16) ;(\text { Hong } \\
\text { Kong: } N=225 ; \text { Australia: } N= \\
\text { 1752); Trauma registry; } \\
\text { Prospective multicentre study }\end{array}$ & $\begin{array}{l}\text { SF-12 } \\
\text { GOSE }\end{array}$ & $\begin{array}{l}6 \text { months (HK: } \\
\text { 72.4\%; Australia: } \\
\text { 83.4\%) } \\
\text { 12 months (HK: } \\
\text { 62.1\%; Australia: } \\
\text { 85.8\%) }\end{array}$ & $\begin{array}{l}\text { Sex, age, ISS, Glasgow Coma } \\
\text { Scale }\end{array}$ & $\begin{array}{l}\text { PCS: } \\
-6 \text { months: HK: } 42.7 \\
\text { (9.8); AUS: } 41.6(11.8) \\
\text { - } 12 \text { months: HK: } 42.2 \\
\text { (11.0); AUS: } 42.6 \text { (12.0) } \\
\text { MCS: } \\
\text { - } 6 \text { months: HK: } 51.8 \\
\text { (12.4); AUS: } 50.6(11.4) \\
\text { - } 12 \text { months: HK: } 52.2 \\
\text { (10.9); AUS: } 50.3(11.2)\end{array}$ \\
\hline $\begin{array}{l}\text { Rainer, } 2014[55], \\
\text { Hong Kong }\end{array}$ & $\begin{array}{l}\text { Adult }(\geq 18) \text { patients } \\
\text { moderate/major trauma } \\
(\text { ISS } \geq 9)(N=400) ; \\
\text { Prospective multicentre }\end{array}$ & $\begin{array}{l}\text { SF-36 } \\
\text { GOSE }\end{array}$ & $\begin{array}{l}\text { Baseline } \\
\text { (preinjury) } \\
\text { Discharge-30 } \\
\text { days }(84 \%) 6\end{array}$ & $\begin{array}{l}\text { Age }>65 \text {, male, pre-injury } \\
\text { health problems, admission } \\
\text { to ICU, ISS, baseline, } 1 \text { and } \\
6 \text { month PCS, } 6 \text { month MCS }\end{array}$ & $\begin{array}{l}\text { GOSE: Upper good } \\
\text { recovery \%: } \\
\text { - Baseline: } 3.5 \% \\
\text { - } 1 \text { month: } 9.7 \%\end{array}$ \\
\hline
\end{tabular}


Table 1 Study characteristics of included articles measuring HRQL in general injury populations (Continued)

\begin{tabular}{|c|c|c|c|c|c|}
\hline $\begin{array}{l}\text { Author, year, } \\
\text { country }\end{array}$ & Study population and design & HRQL instrument & $\begin{array}{l}\text { Follow up time } \\
\text { points }\end{array}$ & Predictors of HRQL/Disability & Outcomes \\
\hline & study & & $\begin{array}{l}\text { months }(70 \%) 12 \\
\text { months }(59 \%)\end{array}$ & (univariate analysis only) & $\begin{array}{l}\text { - } 6 \text { months: } 16.0 \% \\
\text { - } 12 \text { months: } 16.5 \% \\
\text { HRQL: } \% \text { above norm } \\
\text { PCS: (norm HK: } 52.83 \text { ) } \\
\text { - Baseline: } 4.8 \% \\
\text { - } 1 \text { month: } 6.7 \% \\
\text { - } 6 \text { months: } 15.0 \% \\
-12 \text { months: } 15.5 \% \\
\text { MCS: (norm HK: } \\
47.18 \text { ) } \\
\text { - Baseline: } 57.0 \% \\
\text { - } 1 \text { month: } 28.5 \% \\
\text { - } 6 \text { months: } 39.7 \% \\
\text { - } 12 \text { months: } 31.2 \%\end{array}$ \\
\hline $\begin{array}{l}\text { Ringdal, } 2010 \\
\text { [56], Sweden }\end{array}$ & $\begin{array}{l}\text { Adult injury patients that } \\
\text { required intensive care }(N= \\
344) ; \text { Hospital; Prospective } \\
\text { multicentre study }\end{array}$ & SF-36 & $\begin{array}{l}4.5 y \text { to } 5.5 y \text { after } \\
\text { injury (71\%) }\end{array}$ & $\begin{array}{l}\text { Delusional memories during } \\
\text { ICU stay, pre-existing disease } \\
\text { prior trauma }\end{array}$ & $\begin{array}{l}\text { 0.5-1.5 years: } \\
\text { - PCS: } 65.9(31.6) \\
\text { - MCS: } 63.7(27.3) \\
\text { 4.5-5.5 years: } \\
\text { - PCS: } 71.9(30.1) \\
\text { - MCS: } 71.2(22.5)\end{array}$ \\
\hline $\begin{array}{l}\text { Rivara, } 2014[57], \\
\text { US }\end{array}$ & $\begin{array}{l}\text { Trauma patients (parents \& } \\
\text { children), with only parent } \\
\text { injured, only child injured, } \\
\text { both injured or neither } \\
\text { injured }(N=570) \text {; Medical } \\
\text { Centre; Prospective cohort } \\
\text { study }\end{array}$ & $\begin{array}{l}\text { SF-36 (injured) } \\
\text { SF-12 (non- } \\
\text { injured) }\end{array}$ & $\begin{array}{l}5 \text { months } \\
12 \text { months (34\%) }\end{array}$ & $\begin{array}{l}\text { Parents injury affects child } \\
\text { HRQL }\end{array}$ & $\begin{array}{l}\text { Baseline HRQL: } \\
\text { PCS: } \\
\text { Both injured: } 55.5 \\
(9.4) \\
\text { Child injured: } 52.0 \\
\text { (8.2) } \\
\text { Parent injured: } 54.8 \\
(9.1) \\
\text { Neither injured: } 53.2 \\
\text { (8.5) } \\
\text { MCS: } \\
\text { Both injured: } 55.3 \\
(8.3) \\
\text { Child injured: } 51.6 \\
\text { (7.9) } \\
\text { Parent injured: } 54.0 \\
(9.0) \\
\text { Neither injured: } 49.9 \\
(11.2)\end{array}$ \\
\hline $\begin{array}{l}\text { Schneeberg, } \\
2016 \text { [58], British } \\
\text { Columbia }\end{array}$ & $\begin{array}{l}\text { Children (0-16y) who } \\
\text { presented with primary injury } \\
\text { at British Columbia Children's } \\
\text { Hospital }(N=582) \text {; } \\
\text { Prospective cohort study }\end{array}$ & $\begin{array}{l}\text { PedsQL } 4.0 \\
\text { Generic Core } \\
\text { PedsQL infant } \\
\text { scales }\end{array}$ & $\begin{array}{l}\text { Pre-injury (+ at } \\
\text { least } 1 \text { follow-up: } \\
35 \%) \\
1 \text { month (44\%) } \\
4-6 \text { months (29\%) } \\
12 \text { months (28\%) }\end{array}$ & $\begin{array}{l}\text { Greater impact on HRQL } 1 \\
\text { month post injury, steeper } \\
\text { slope to recovery: Older age, } \\
\text { hospitalisation }\end{array}$ & $\begin{array}{l}\text { Mean HRQL: } \\
\text { - Baseline: } 90.7 \\
\text { - } 1 \text { month: } 77.8 \\
\text { - } 4 \text { months: } 90.3 \\
\text { - } 12 \text { months: } 91.3\end{array}$ \\
\hline $\begin{array}{l}\text { Soberg, } 2012 \\
\text { [59], Norway }\end{array}$ & $\begin{array}{l}\text { Patients } 18-67 y \text { with an } \\
\text { NISS } \geq 16 \text { and at least } 2 \text { in- } \\
\text { juries classified in AIS ( } N= \\
\text { 105); University hospital; } \\
\text { Prospective cohort study }\end{array}$ & $\begin{array}{l}\text { SF-36 } \\
\text { WHODAS II }\end{array}$ & $\begin{array}{l}6 \text { weeks } \\
1 \text { year }(99 \%) \\
2 \text { years }(94 \%) \\
5 \text { years }(80 \%)\end{array}$ & $\begin{array}{l}\text { PCS: Time points of } \\
\text { measurement, time in } \\
\text { hospital/rehabilitation, } \\
\text { getting around, } \\
\text { participation in society } \\
\text { MCS: time points of } \\
\text { measurement, sex, } \\
\text { education, WHODAS II } \\
\text { cognitive function \& } \\
\text { participation in society }\end{array}$ & $\begin{array}{l}\text { WHODAS-II scores: } \\
\text { Understanding/ } \\
\text { communicating: } \\
\text { - } 6 \text { weeks: } 10.0(0.0- \\
30.0) \\
-1 \text { year: } 10.0(0.0- \\
25.0) \\
-2 \text { years: } 10(0.0-25.0) \\
-5 \text { years: } 10.0(0.0- \\
30.0) \\
\text { Getting around: } \\
-6 \text { weeks: } 37.5(12.5- \\
62.5) \\
-1 \text { year: } 12.5(0.0- \\
37.5) \\
-2 \text { years: } 12.5(0.0- \\
37.5) \\
-5 \text { years: } 12.5(0.0- \\
31.3) \\
\text { Self-care: }\end{array}$ \\
\hline
\end{tabular}


Table 1 Study characteristics of included articles measuring HRQL in general injury populations (Continued)

Author, year, Study population and design HRQL instrument Follow up time Predictors of HRQL/Disability
country points

\begin{tabular}{|c|c|}
\hline $\begin{array}{l}\text { Soberg, } 2015 \\
\text { [60], Norway }\end{array}$ & $\begin{array}{l}\text { Patients (18-67y) with } \\
\text { severe multiple injuries } \\
(N=105) ; \text { Hospital; } \\
\text { Prospective cohort study }\end{array}$ \\
\hline $\begin{array}{l}\text { Tamura, } 2018 \\
\text { [61], Japan }\end{array}$ & $\begin{array}{l}\text { All eligible consecutive } \\
\text { trauma patients admitted to } \\
\text { the intensive care unit of one } \\
\text { tertiary care hospital ( } N= \\
\text { 187); Prospective cohort } \\
\text { study }\end{array}$ \\
\hline
\end{tabular}

$\begin{array}{ll}\text { SF-36 } & 1 \text { year } \\ & 2 \text { years } \\ & 5 \text { years } \\ & 10 \text { years }(55.2 \%)\end{array}$

PCS: change in coping from 2 to 10 years

PCS and MCS: bodily pain at 2 years;

MCS: change in coping, vitality at 1 year, social functioning and mental health at 2 years

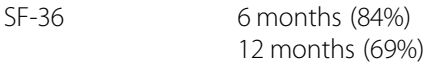

lower age, head injury;

\author{
- 6 weeks: 20.0 (0.0- \\ 30.0) \\ - 1 year: $0.0(0.0-10.0)$ \\ - 2 years: $0.0(0.0-$ \\ 10.0) \\ - 5 years: $0.0(0.0-$ \\ 10.0) \\ Getting along with \\ people: \\ - 6 weeks: 16.7 (8.3- \\ 35.4) \\ - 1 year: $16.7(0.0-$ \\ 25.0) \\ - 2 years: 16.7 (8.3- \\ 33.3) \\ - 5 years: 20.8 (8.3- \\ 33.3) \\ Life activities: \\ - 6 weeks: 50.0 (35.0- \\ 80.0) \\ - 1 year: 30.0 (10.0- \\ 50.0) \\ - 2 years: $40.0(0.0-$ \\ 50.0) \\ - 5 years: $20.0(0.0$, \\ 50.0) \\ Participation in \\ society: \\ - 6 weeks: 45.8 (37.5- \\ 58.3) \\ - 1 year: 25.0 (12.5- \\ 41.7) \\ - 2 years: 25.0 (8.3- \\ 41.7) \\ - 5 years: 18.8 (8.3- \\ 34.4)
}

10 years:

- PCS: 41.8 (11.7)

- MCS: 48.8 (10.7)

Reduced PCS

compared with adjusted general population; MCS not different from general population

Median [IQR]:

- Discharge: PCS: 21 [10,

35]; MCS: 56 [48, 66]

- 6 months: PCS: 43 [33,

51]; MCS: 52 [44, 61]

- 12 months: PCS: 44

[32, 53]; MCS: 53 [46,

59]

Role Social:

- Discharge: 21 [10, 38]

- 6 months: 39 [23, 52]

- 12 months: 45 [29, 53]

12 months post injury:

$12 \%$ dependent on

home care

HRQL: differences

men/women

3 months:

- Mental health: Men:

76.6; Women: 71.3

Mental functioning: high

depression score baseline, 
Table 1 Study characteristics of included articles measuring HRQL in general injury populations (Continued)

Author, year, Study population and design HRQL instrument Follow up time Predictors of HRQL/Disability Outcomes

country points

higher age, being employed Women: 46.6 or studying before trauma; 12 months:

Bodily pain \& vitality: high - Vitality: Men: 56.8; depression score baseline; Women: $\mathbf{5 0 . 0}$ General health: optimism, low PTSD at baseline, lower ISS

$\begin{array}{ll}\text { Yiengprugsawan, } & \text { Distance learning students } \\ 2014 \text { [63], } & \text { 15-87y enrolled at Sukhothai } \\ \text { Thailand } & \text { Thammathirat Open Univer- } \\ & \text { sity }(N=87,134) ; \text { Prospective } \\ & \text { cohort study }\end{array}$

MOS-SF-8

4 years (70\%)

Injury exposure

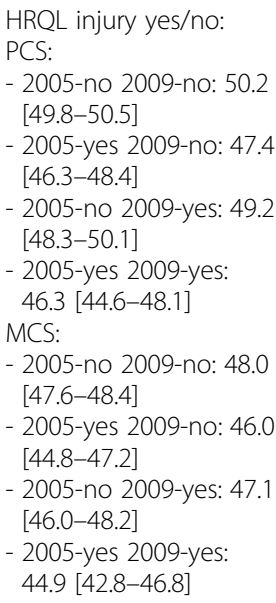

1 month (93\%)

2 months $(82 \%)$ Trauma centre; Prospective cohort study
4 months $(70 \%)$

12 months (58\%)
3 PCS trajectories, 5 MCS trajectories:

PCS: 1. Low baseline score,

no improvement; 2.

Declines 1 month after injury, then improves over time; 3.Sharp decline followed by rapid recovery; MCS 1. Low baseline, remain low; 2. Large decrease post-injury, no recovery over next 12 months; 3.initial decrease in MCS early, followed by continuous recovery; 4 . Steady decline over study period; 5 . Consistently high at all time points

Baseline (preinjury) 1 month (94\%)

2 months (83\%)

4 months (69\%)

12 months (64\%)
PCS: individual income; MCS: high resiliency score; age; income
Different trajectories of recovery

- Either improvement of physical and/or mental health or decline

ISS Injury Severity Score, SF-12 Medical Outcome Study Short Form-12 items, GOSE Extended Glasgow Outcome Scale, SF-36 Medical Outcome Study Short Form36 items, ICU Intensive Care Unit, PCS Physical Component Score, MCS Mental Component Score, EQ-5D-3L EQ-5D with three response options per dimension, GOS Glasgow Outcome Scale, HUI3 Health Utilities Index 3, PTSD Post-traumatic Stress Disorder, MOS-SF-8 Medical Outcome Study Short-Form, ED Emergency Department, ACC Accident Compensation Corporation, QALY Quality Adjusted Life Year, WHODAS I/ World Health Organization Disability Assessment Schedule version II, BMI Body Mass Index, NISS New Injury Severity Score, MFA Musculoskeletal Functional Assessment, TOP Trauma Outcome Profile, AIS Abbreviated Injury Scale, SOFA Sequential Organ Failure Assessment, APACHE-Il Acute Physiology Age Chronic Health Evaluation, ED-HDU Emergency Department High Dependency Unit, RTW Return To Work, EQ-VAS European Quality of Life instrument Visual Analogue Scale, FIM Functional Independence Measure, PedsQL Paediatric Quality of Life Inventory Generic Core Scales

Articles are ordered alphabetically, and articles that come one after the other and have the same bold/non-bold font are from the same study

27-37]. Seven articles were published using data from Australia [24-26, 39-42], with two articles related to the same study cohort from Victoria [41, 42] and two articles related to the same cohort from South-East
Queensland [25, 26]. Five articles reported on five unique studies conducted in the United States [38, 53, $57,64,65]$. Three articles resulted from two studies in Switzerland [43-45] and three articles resulted from two 
studies in Norway [59, 60, 62], respectively. Two articles from two different studies were detected from both Italy $[46,47]$ and Sweden [52, 56]. Remaining articles were from studies conducted in Hong Kong [55], India [48], British Colombia [58], Iran [23], Spain [50], United Kingdom [49], Thailand [63], Japan [61] and Vietnam [51] (all $n=1)$. One study was a multicentre study, conducted in both Australia and Hong Kong [54]. The sample sizes for each investigation ranged from 105 to 87, 134, with the majority of the samples in the range of 105 to 668 participants $(n=28)$. Four studies measured HRQL in children and adolescents $[48,53,57,58]$, while all other studies focussed on adult populations. All studies included a non-specific injury population, with differing injury severities.

Approximately a third $(n=10)$ of all studies focused on all injury severities, with a main inclusion criteria of hospital admission or injuries likely to result in insurance claims for more than just medical treatment. The second largest group of studies focussed on major injuries $(n=18)$. Inclusion criteria were varying, with some studies only requiring $\geq 24 \mathrm{~h}$ stay at the hospital or admission to intensive care unit (ICU) $(n=7)$, and other studies requiring a minimum score on the ISS (Injury Severity Score) or NISS (New Injury Severity Score). ISS for major injuries ranged from ISS $>12(n=2)$ to ISS $\geq 16(n=2)$, versus NISS ranging from NISS $\geq 8(n=1)$ to NISS $\geq 16(n=2)$. The remaining 5 studies focused on moderate $(n=3)$ or mild to moderate $(n=2)$ injuries, with moderate injury studies requiring AIS (Abbreviated Injury Scale) $\geq 2(n=1)$ or ISS $\geq 9(n=2)$, and mild to moderate injury studies requiring ISS $<15(n=1)$ and length of hospitalisation $<24 \mathrm{~h}(n=1)$.

\section{Study design}

All studies that were included in this review were prospective cohort studies. Seven out of the 29 unique studies were multicentre studies [24, 48, 49, 52, 54-56]. Across studies HRQL and disability were measured with 14 different measurement instruments. Generic instruments SF-36 $(n=13)$ and EQ-5D $(n=7)$ were most commonly used, followed by SF-12 $(n=6)$ and GOSE $(n=4)$, as can be retrieved from Fig. 2. Approximately $45 \%$ of the studies $(n=13)$ used more than one measurement instrument, of which 10 used two instruments, and 3 used more than two instruments. All measurement instruments were generic, with three out of four studies in children using a child-specific instrument (PedsQL; PedsQL 4.0; PedsQL infant scales) only, and one study in children using two all ages instruments (SF-12 and SF-36). Measurement of HRQL was conducted at different time points in studies, with the number of follow-up points varying from one $(n=4)$ to five $(n=3)$. HRQL was assessed at more than one follow-up point in 25 studies, with measurement at 6 and 12 months most frequent across all studies ( $n=14$ and $n=19$, respectively) (Fig. 3). Three other common measurement points were 24 months $(n=12), 1$ month $(n=9)$ and 3 months $(n=$ 7) after injury. Studies used different administration methods of questionnaires, with telephone interview as the most common method $(n=13)$. A combination of different methods was common, with baseline measurement often performed in a face-to-face interview, and later follow-up measurements done by either telephone or postal/email interview.

\section{Quality of studies}

Length of follow-up was consistent for all study participants in all but two studies $[25,26,56]$. The same results were found regarding whether follow-up time was sufficient for measuring primary outcomes, with only two studies reporting an insufficient follow-up period [24, 47]. However, attrition appeared to be a problem in many studies: 18 out of 29 studies exceeded the attrition

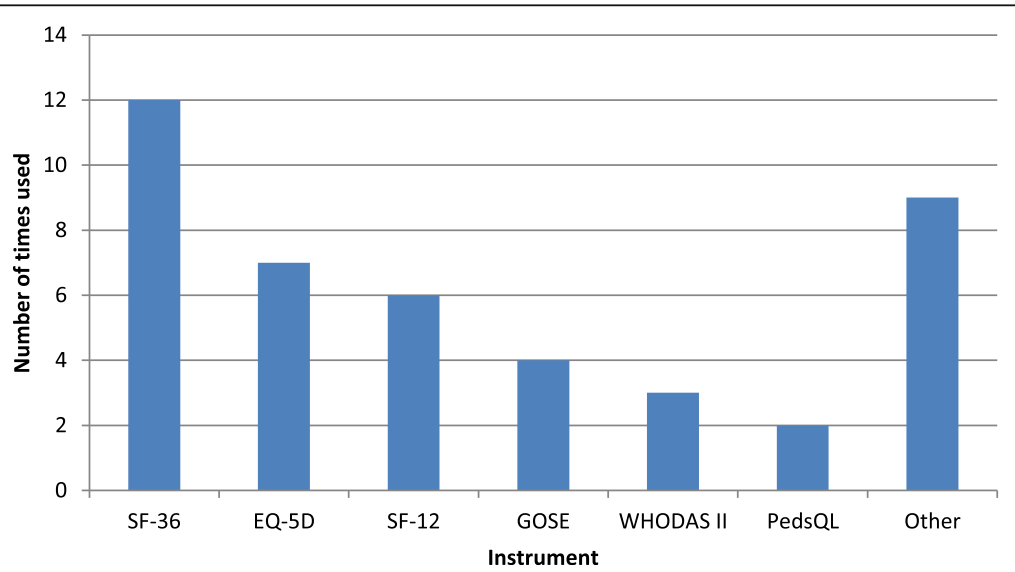

Fig. 2 Frequency of generic measures used in studies to assess HRQL. Note1: Some studies used more than 1 measurement instrument. Note2: 'Other' consists of: GOS (2), HUI3 (1), MOS-SF-8 (1), MFA (1), TOP (1), FIM (1), PedsQL 4.0 Generic core (1), PedsQL infant scales (1) 


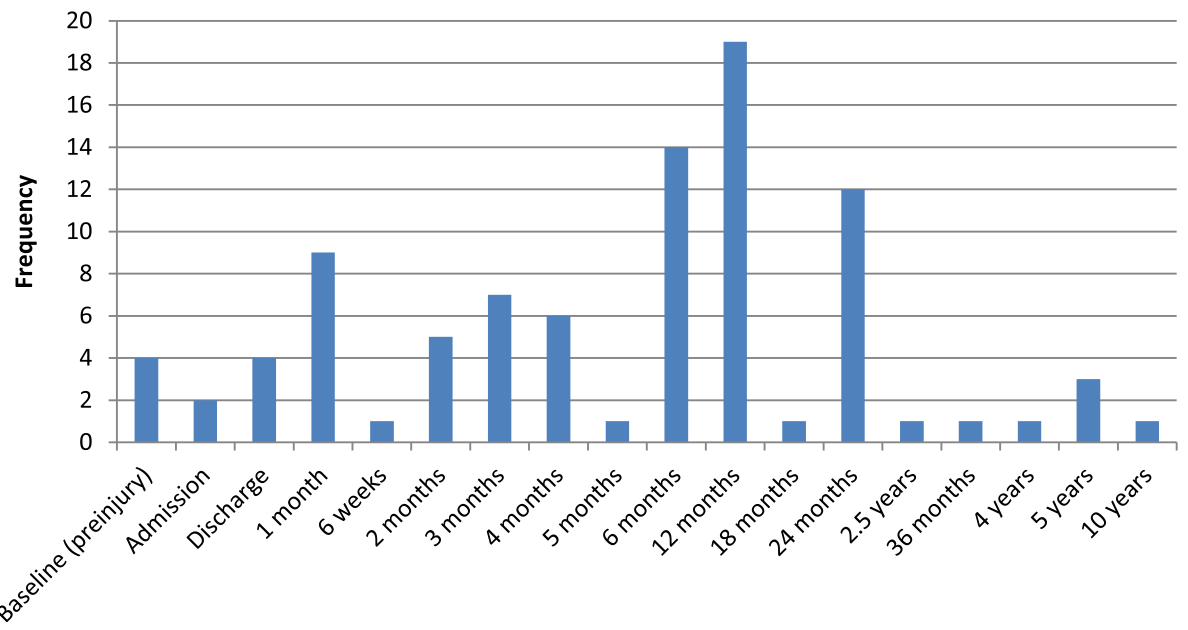

Fig. 3 Frequency of time points at which HRQL was measured across studies

norm of $20 \%$ for $<1$ year follow-up and $30 \%$ for $\geq 1$ year follow-up.

Regarding adherence to the Guidelines for the Conduction of Follow-up Studies Measuring Injury-Related Disability, it was found that study populations were generally in accordance with the guidelines. However, measurement in respondents with mental and/or social problems was only specifically mentioned in two studies $[40,48]$, whereas all other studies provided no or unclear information on the subject. Even though the guidelines recommend a combination of the EQ-5D and HUI3 to measure HRQL, none of the included studies used this combination. The EQ-5D and HUI3 were used separately in a number of studies $[14,30-35,39,42-45,49-$ 51]. Six studies complied to the measurement points required by the guidelines, namely one, two, four and 12 months after injury [48, 49, 51, 58, 64, 65]. Even though other studies did not follow all required measurement points, the majority complied with at least one.

\section{Predictors for HRQL}

Recovery patterns of HRQL after injury were found to differ across subgroups in most studies. There was substantial variation in the predictors of HRQL after injury, however, seven predictors were mentioned in six or more articles: age $(n=14)$, gender $(n=12)$, pre-injury health status $(n=12)$, hospitalisation status $(n=7)$, nature of injury $(n=7)$, injury severity $(n=7)$ and socioeconomic status $(n=6)$. Older age and female gender were found to have a negative impact on the outcome of HRQL after trauma in several articles [24, 31, 41, 47, 50, $51]$, whereas in two other articles male gender was found to have a negative association with $\operatorname{HRQL}[45,55]$.

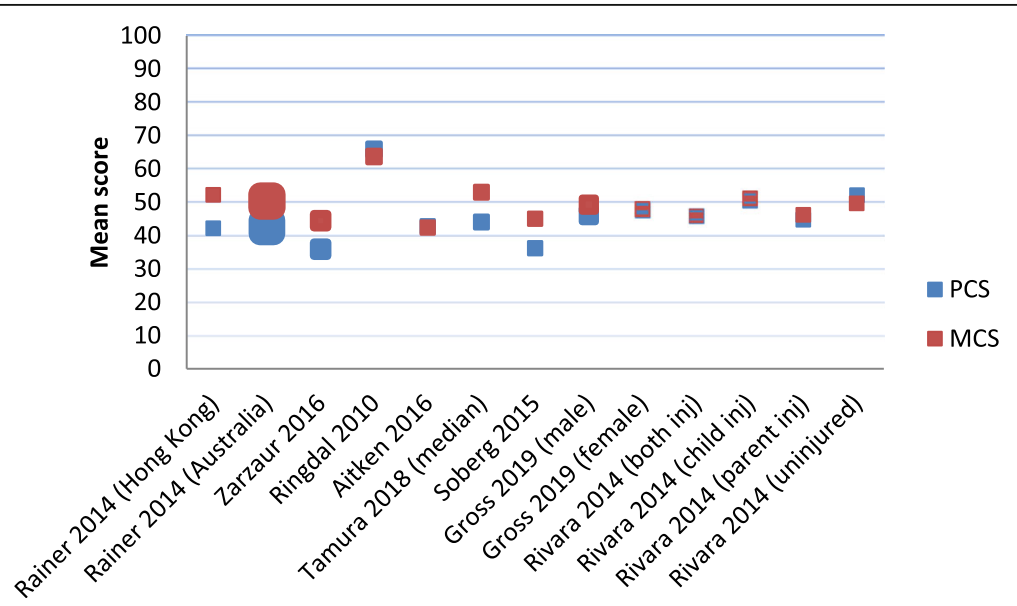

Fig. 4 SF-12 and SF-36 scores at 12 months after injury. Note1: The $y$-axis shows the mean scores, not utility values. Note2: The size of the dots is proportional to the sample size 


\section{Changes over time}

Studies that reported HRQL values generally reported improvements in HRQL over time (see Table 1). However, not all studies that were included reported specific outcomes of HRQL, as some studies reported on odds ratio and relative risks. Improvement in HRQL was found in all studies, however, pre-injury status or population level was not reached for the total injury population after 6-24 months [24, 26, 31, 36, 44, 46, 47, 49, 55, $60,62]$. Figures 4 and 5 summarise HRQL scores of all articles that provided a mean HRQL score at 12 months after injury. Some articles provided mean scores only per subgroup, and have therefore been included in the figure for each subgroup. Figure 4 shows the physical component score (PCS) and mental component score (MCS) for both SF-12 and SF-36, whereas Fig. 5 shows the summary score for the EQ-5D, EQ-VAS, HUI3 and PedsQL (4.0).

\section{Discussion}

This systematic review aimed to provide an update on studies measuring HRQL with a generic instrument in general injury populations since the publication of an earlier review examining injury studies conducted between 1995 and 2009 [12]. Given the increase in the number of studies conducted in this area over recent years, our review focused specifically on studies that examined HRQL at more than one time point. As with the earlier review, considerable methodological variation across studies was found; differences were apparent in study settings, injury severity of participants, HRQL instruments used, follow-up periods, and timing of HRQL assessments. The most commonly used instruments to assess HRQL included the SF-36, SF-12, and EQ-5D, although 14 different instruments were applied across the
29 studies included in this review. Study follow-up points ranged from 1 month to 10 years post-injury, with follow-up assessments most commonly occurring at 6 , 12 and 24 months after injury.

Despite the variation across studies included in this review, it is important to note that improvement in the consistency of study designs was observed since the earlier review of studies measuring HRQL in general injury populations [12]. Our review found a greater number of studies that had employed a longitudinal design over a shorter review period; we identified 29 longitudinal studies over a 9 year period in contrast to the 21 longitudinal studies published across the 14 years examined by Polinder et al. Our updated review also found that longer durations of follow-up have been utilised, with four studies examining HRQL beyond 24 months, and one up to 10 years post-injury. This is in contrast to the earlier review where many studies had examined outcomes until 6 months only, and none had examined outcomes beyond 24 months. These findings demonstrate an increase in adherence to the recommendations of the European Consumer Safety Association [16], which recommends assessments be conducted to a minimum of 12 months post-injury.

While longer follow-up periods are occurring in studies examining $\mathrm{HRQL}$ in general injury populations, the timing of assessments continues to vary across studies. The 2007 guidelines recommend assessments at regular intervals of 1,2, 4 and 12 months post-injury, allowing for examination of the four phases of trauma recovery: acute treatment phase, rehabilitation phase, adaptation phase, and stable end situation [16]. Only five studies completed follow-ups at these time points $[48,49,51$, $64,65]$, although five completed assessments at four different times in the 12 months after injury [50, 53, 58],

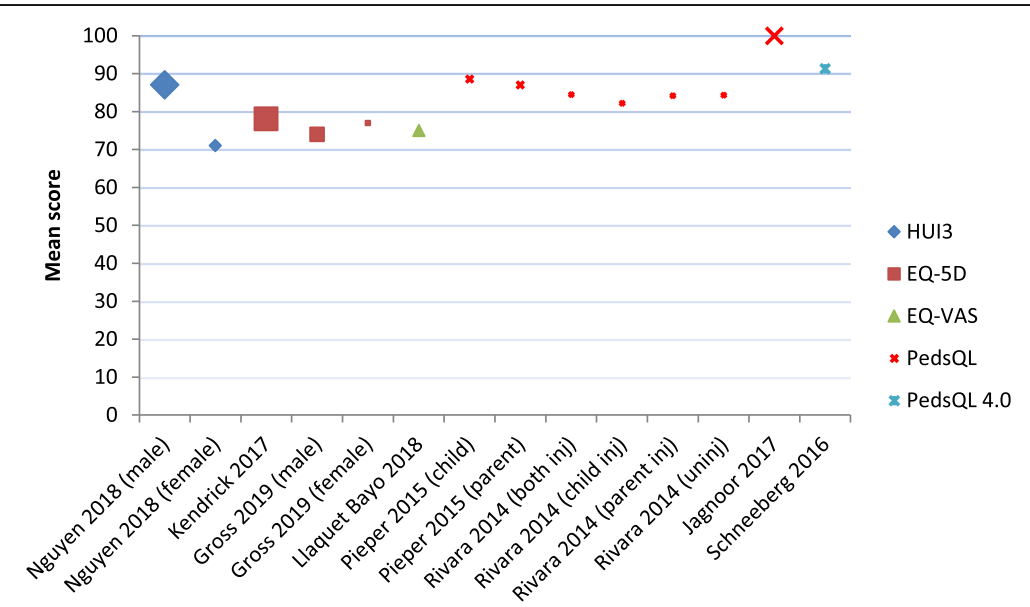

Fig. 5 EQ-5D, PedsQL (4.0), HUI3 and EQ-VAS scores at 12 months after injury. Note1: The y-axis shows descriptive summary scores only, not utility values. Scores are not directly comparable due to the different HRQL measures used. Note2: Scale from 0 to 100 for PedsQL (4.0) and EQVAS; scale from 0 to 1 for EQ-5D and HUI3 (score multiplied by 100). Note3: The size of the dots is proportional to the sample size of the study 
and five examined outcomes at least four times over a longer period (beyond 12 months) [26, 40, 42]. There may be important reasons for researchers selecting different times of outcome assessment than those recommended. For example, examination beyond the 12 month point is likely to be important given accumulating evidence that changes (including improvements and deteriorations) in health status can continue to be detected after this time [59, 60]. Ensuring that participant burden is kept to a minimum is likely to be another important consideration.

Guidelines for the examination of health status among injury populations also recommend the inclusion of a retrospective recalled assessment of pre-injury health $[16,66]$. Few studies in our review met this criteria, despite evidence that such retrospective measurements are likely to be more appropriate than comparisons with general population norms when evaluating post-injury losses in HRQL $[9,67]$. This is because individuals from the general population are unlikely to be representative of those from an injured population [68]. A systematic review of studies collecting pre-injury HRQL data among injury patients has demonstrated that both general population comparisons and retrospective assessments are likely to result in biased estimates of preinjury HRQL [69]. However, prospective HRQL data is often impractical to collect prior to an injury occurring. Instead, it may be most feasible to collect retrospective assessments of pre-injury HRQL as soon as practicably possible after injury.

The identification of 14 different instruments to evaluate HRQL across the 29 studies included in this updated review suggests that there remains significant variation in the types of measures used. However, it is important to recognise that this variation has decreased substantially since the earlier systematic review of studies evaluating HRQL after injury, from which 24 different generic HRQL and functional status measures were extracted. This indicates that the potential to make comparisons across studies is increasing. While a number of studies employed the EQ-5D in isolation, no studies used both the EQ-5D and the HUI3 to evaluate HRQL, which is recommended in the guidelines [16]. Many studies used neither the EQ-5D nor the HUI3, instead employing the SF-12 or SF-36 to assess HRQL. Understanding motivations behind the selection of instruments to examine HRQL and disability outcomes after injury is an important avenue for future research. Different outcome measures focus more or less on specific HRQL dimensions and the dimensions of interest to researchers may vary across countries depending on the aspects of health that are most relevant to each unique social, cultural, and political context.
Included studies varied in the reporting of HRQL information. While some studies reported the proportion of people experiencing problems with particular HRQL and disability domains others reported summary or utility scores. The 14 studies included in the review reporting summary scores represents only a slight increase from the 12 studies that did so in the earlier review.

As with the earlier review, our review found that generic instruments are capable of detecting changes in HRQL between discharge and follow-up. Despite continuing variation in study design, it is evident that the greatest gains in health status are observed in the first 12 months after injury. Gains can also be observed in the following 12 months (up to 24 months post-injury) among individuals who have sustained serious injuries (as indicated by injury severity scores and hospitalisation status). Although these gains can be detected, many studies concluded that HRQL remains significantly reduced in comparison to pre-injury levels or population norms, and this is evident up to 10 years after injury [60]. While these insights are important, continued variation in assessment time points, study populations, HRQL instruments, and the reporting of HRQL outcomes makes it difficult to compare findings from individual studies, and reduces the precision of knowledge regarding the global impact of injury on population health over time.

An important limitation associated with this systematic review is that only peer-reviewed published literature was included. It is possible that other longitudinal studies examining HRQL in large injury populations have been conducted but not published. Another limitation is that studies that examined HRQL or disability were eligible for inclusion in the review, and although these constructs are related, they are not synonymous. Despite these limitations, the review provides important insight into the design and findings of studies published since 2010. The variation observed across included studies suggests that the European Consumer Safety Association guidelines for the conduction of follow-up studies may be difficult for researchers to adhere to. Further research is needed to explore the reasons why researchers are not following these guidelines. This information could be used to inform the development of updated guidelines that are feasible to follow when taking into account the significant contextual variation that exists across different countries and populations. This, in turn, may lead to increased consistency in study designs and outcome reporting, allowing for meaningful crosscountry comparisons.

\section{Conclusions}

Although increased consistency in studies designed to investigate $\mathrm{HRQL}$ in general injury populations has been 
observed since 2010, there remains significant variation that makes comparisons across studies difficult and prevents precise estimates of the impact of injury on global health. Exploring reasons for variation in study design and reporting of outcomes is an important avenue for future research that may inform the development of updated guidelines for the conduct of follow-up studies measuring HRQL and disability outcomes among individuals with injury.

\section{Appendix}

\section{Search strategies}

Embase.com:

('quality of life'/exp. OR 'quality of life assessment'/exp. OR 'health status indicator'/de OR 'life satisfaction'/de OR 'functional status assessment'/de OR 'Functional Assessment Inventory'/de OR 'Functional Independence Measure'/de OR 'Health Assessment Questionnaire'/de OR ('health status'/de AND 'rating scale'/de) OR (((quality OR satisf") NEAR/3 (life OR wellbeing OR well-being)) OR hrql OR hrqol OR (('health status' OR disabilit* OR functional"-independen* OR Functional-Assess* OR Functional-status* OR Functioning OR sickness-impact OR health-utilit*) NEAR/3 (indicator* OR eval* OR assess* OR measure* OR profile* OR index" OR Classification")) OR (('Short Form' OR SF) NEXT/1 (36 OR 20 OR 12 OR 6)) OR sf36 OR sf20 OR sf12 OR sf6 OR health-profile* OR euroqol OR eq-5d OR hui-2 OR hui2 OR hui-3 OR hui3 OR QWB OR WHODAS-II OR WHODAS-2 OR who-das-ii OR who-das-2):ab,ti) AND ('injury'/de OR 'childhood injury'/de OR 'injury severity'/de OR 'accidental injury'/de OR 'injury scale'/de OR 'multiple trauma'/de OR (injur" OR trauma"):ab,ti) AND ('cohort analysis'/de OR 'longitudinal study'/de OR 'prospective study'/de OR 'retrospective study'/de OR (cohort" OR longitudinal* OR prospectiv* OR retrospectiv*):ab,ti) NOT ([Conference Abstract]/lim) AND [English]/lim AND [2010-2018].

Medline Ovid:

(Quality of Life/ OR Health Status Indicators/ OR Disability Evaluation/ OR (((quality OR satisf*) ADJ3 (life OR wellbeing OR well-being)) OR hrql OR hrqol OR ((health status OR disabilit* OR functional"-independen* OR Functional-Assess" OR Functional-status* OR Functioning OR sickness-impact OR health-utilit*) ADJ3 (indicator* OR eval* OR assess* OR measure* OR profile* OR index* OR Classification*)) OR ((Short Form OR SF) ADJ (36 OR 20 OR 12 OR 6)) OR sf36 OR sf20 OR sf12 OR sf6 OR health-profile* OR euroqol OR eq-5d OR hui-2 OR hui2 OR hui-3 OR hui3 OR QWB OR WHODAS-II OR WHODAS-2 OR who-das-ii OR whodas-2).ab,ti.) AND ("Wounds and Injuries"/ OR Injury Severity Score/ OR Multiple Trauma/ OR (injur* OR trauma*).ab,ti.) AND (exp Cohort Studies/ OR (cohort* OR longitudinal* OR prospectiv* OR retrospectiv*).ab,ti.) AND english.la.
Limit 2010-2018.

PsycINFO Ovid:

("Quality of Life"/ OR Disability Evaluation/ OR (((quality OR satisf*) ADJ3 (life OR wellbeing OR wellbeing)) OR hrql OR hrqol OR ((health status OR disabilit" OR functional"-independen* OR FunctionalAssess* OR Functional-status* OR Functioning OR sickness-impact OR health-utilit*) ADJ3 (indicator* OR eval* OR assess* OR measure* OR profile* OR index* OR Classification*)) OR ((Short Form OR SF) ADJ (36 OR 20 OR 12 OR 6)) OR sf36 OR sf20 OR sf12 OR sf6 OR health-profile* OR euroqol OR eq-5d OR hui-2 OR hui2 OR hui-3 OR hui3 OR QWB OR WHODAS-II OR WHODAS-2 OR who-das-ii OR who-das-2).ab,ti.) AND ("Injuries"/ OR (injur* OR trauma*).ab,ti.) AND (Cohort Analysis/ OR Longitudinal Study.md. OR Prospective Study.md. OR Retrospective Study.md. OR (cohort* OR longitudinal* OR prospectiv* OR retrospectiv*).ab,ti.) AND english.la.

Limit 2010-2018.

Web of science:

TS $=(((($ quality OR satisf*) NEAR/2 (life OR wellbeing OR well-being)) OR hrql OR hrqol OR (("health status" OR disabilit* OR functional*-independen* OR Functional-Assess* OR Functional-status* OR Functioning OR sickness-impact OR health-utilit*) NEAR/2 (indicator* OR eval" OR assess" OR measure* OR profile* OR index" OR Classification*)) OR (("Short Form” OR SF) NEAR/1 (36 OR 20 OR 12 OR 6)) OR sf36 OR sf20 OR sf12 OR sf6 OR health-profile* OR euroqol OR eq5d OR hui-2 OR hui2 OR hui-3 OR hui3 OR QWB OR WHODAS-II OR WHODAS-2 OR who-das-ii OR whodas-2)) AND ((injur* OR trauma*)) AND ((cohort* OR longitudinal* OR prospectiv* OR retrospectiv*))) AND $\mathrm{DT}=($ article $)$ AND LA $=$ (english)

Limit 2010-2018.

\section{Abbreviations}

AIS: Abbreviated Injury Scale; FIM: Functional Independence Measure; GOS: Glasgow Outcome Scale; GOSE: Extended Glasgow Outcome Scale; HRQL: Health-Related Quality of Life; HUI 3: Health Utilities Mark III; ICF: World Health Organization International Classification of Functioning, Disability and Health; ICU: Intensive Care Unit; ISS: Injury Severity Score; MCS: Mental Component Score; NISS: New Injury Severity Score; PCS: Physical Component Score; PedsQL: Paediatric Quality of Life Inventory Generic Core Scales; SF12: Medical Outcome Study Short Form-12 items; SF-36: Medical Outcome Study Short Form-36 items; WHO: World Health Organization; WHODAS: World Health Organization Disability Assessment Schedule

\section{Acknowledgements}

Not applicable.

\section{Authors' contributions}

AG and AR contributed equally to the study in terms of searching studies, analysing studies and summarizing findings. JH, SD and SP overlooked the selection process and substantively revised the manuscript. All authors read and approved the final manuscript.

Funding

No funding was received for this study. 


\section{Availability of data and materials}

Not applicable.

\section{Ethics approval and consent to participate}

Not applicable.

\section{Consent for publication}

Not applicable.

\section{Competing interests}

The authors declare that they have no competing interests.

\section{Author details}

'Department of Public Health, Erasmus MC, University Medical Center Rotterdam, P.O. Box 2040, 3000 Rotterdam, CA, The Netherlands. ${ }^{2}$ Injury Prevention Research Unit, Department of Preventive and Social Medicine, Dunedin School of Medicine, University of Otago, Dunedin, New Zealand.

\section{Received: 3 October 2019 Accepted: 19 May 2020}

\section{Published online: 29 May 2020}

\section{References}

1. GBD 2016 Disease and Injury Incidence and Prevalence Collaborators. Global, regional, and national incidence, prevalence, and years lived with disability for 328 diseases and injuries for 195 countries, 1990-2016: a systematic analysis for the Global Burden of Disease Study 2016. Lancet. 2017:390(10100):1211-59.

2. Michaud CM, Murray $\mathrm{CL}$, Bloom BR. Burden of disease-implications for future research. JAMA. 2001;285(5):535-9.

3. Segui-Gomez M, MacKenzie EJ. Measuring the public health impact of injuries. Epidemiol Rev. 2003;25(1):3-19.

4. National Center for Health Statistics. Medically consulted injury and poisoning episodes, by external cause and selected characteristics. United States; 2014. https://www.cdc.gov/nchs/nhis/SHS/tables.htm. Accessed 4 Dec 2018

5. Üstun TB, Chatterii S, Bickenbach J, Kostanisek N, Schneider M. The international classification of functioning, disability and health: a new tool for understanding disability and health. Disabil Rehabil. 2003;25(11-12):565-71.

6. Haagsma JA, Graetz N, Bolliger I, et al. The global burden of injury: incidence, mortality, disability-adjusted life years and time trends from the global burden of disease study 2013. Injury Prevent. 2016;22(1):3-18.

7. Ware JE. Conceptualization and measurement of health-related quality of life: comments on an evolving field. Arch Phys Med Rehabil. 2003;84:S43-51.

8. Lyons RA, Polinder S, Larsen CF, et al. Methodological issues in comparing injury incidence across countries. Int J Inj Control Saf Promot. 2006;13(2):63-70.

9. Watson WL, Ozanne-Smith J, Richardson J. Retrospective baseline measurement of self-reported health status and health-related quality of life versus population norms in the evaluation of post-injury losses. Injury Prevent. 2007:13(1):45-50.

10. Power M, Bullinger M, Harper A. The World Health Organization WHOQOL 100: tests of the universality of quality of life in 15 different cultural groups worldwide. Health Psychol. 1999;18(5):495-505.

11. Whitehead SJ, Ali S. Health outcomes in economic evaluation: the QALY and utilities. Br Med Bull. 2010;96(1):5-21.

12. Polinder $\mathrm{S}$, Haagsma JA, Belt $\mathrm{E}$, et al. A systematic review of studies measuring health-related quality of life of general injury populations. BMC Public Health. 2010;10(1):783

13. Moriarty DG, Zack MM, Kobau R. The Centers for Disease Control and Prevention's healthy days measures - population tracking of perceived physical and mental health over time. Health Qual Life Outcomes. 2003;1 (1):37.

14. Derrett $S$, Davie G, Ameratunga $S$, et al. Prospective outcomes of injury study: recruitment, and participant characteristics, health and disability status. Injury Prevent. 2011;17(6):415-8.

15. Lyons RA, Kendrick D, Towner EM, et al. Measuring the population burden of injuries-implications for global and national estimates: a multi-Centre prospective UK longitudinal study. PLoS Med. 2011;8(12): e1001140.

16. Van Beeck EF, Larsen CF, Lyons RA, Meerding W-J, Mulder S, Essink-Bot M-L. Guidelines for the conduction of follow-up studies measuring injury-related disability. J Trauma Acute Care Surg. 2007;62(2):534-50.
17. Mokkink $L B$, Terwee $C B$, Patrick DL, et al. The COSMIN checklist for assessing the methodological quality of studies on measurement properties of health status measurement instruments: an international Delphi study. Qual Life Res. 2010;19(4):539-49.

18. World Health Organization. Disabilities. 2019; https://www.who.int/topics/ disabilities/en/. Accessed 26 Aug 2019.

19. Centers for Disease Control and Prevention. Health-Related Quality of Life (HRQOL). 2018; https//www.cdc.gov/hraol/concept.htm. Accessed 26 Aug 2019.

20. Cieza A, Stucki G. Content comparison of health-related quality of life (HRQOL) instruments based on the international classification of functioning, disability and health (ICF). Qual Life Res. 2005;14(5):1225-37.

21. Viswanathan $M$, Berkman ND. Development of the RTI item bank on risk of bias and precision of observational studies. J Clin Epidemiol. 2012;65(2):163-78.

22. World Health Organization. Disability Assessment Schedule II. http://www. who.int/icidh/whodas/. Accessed 7 Jan 2019.

23. Abedzadeh-Kalahroudi M, Razi E, Sehat M, Asadi LM. Measurement of disability and its predictors among trauma patients: a follow-up study. Arch Trauma Res. 2015;4(3):e29393.

24. Aitken LM, Chaboyer W, Kendall E, Burmeister E. Health status after traumatic injury. J Trauma Acute Care Surg. 2012;72(6):1702-8.

25. Aitken LM, Chaboyer W, Schuetz M, Joyce C, Macfarlane B. Health status of critically ill trauma patients. J Clin Nurs. 2014;23(5-6):704-15.

26. Aitken LM, Macfarlane B, Chaboyer W, Schuetz M, Joyce C, Barnett AG. Physical function and mental health in trauma intensive care patients: a 2year cohort study. Crit Care Med. 2016;44(4):734-46.

27. Davie G, Samaranayaka A, Derrett S. The role of pre-existing comorbidity on the rate of recovery following injury: a longitudinal cohort study. PLoS One. 2018:13(2):e0193019.

28. Derrett $\mathrm{S}$, Samaranayaka A, Wilson $\mathrm{S}$, et al. Prevalence and predictors of subacute phase disability after injury among hospitalised and non-hospitalised groups: a longitudinal cohort study. PloS One. 2012;7(9):e44909.

29. Derrett S, Wilson S, Samaranayaka A, et al. Prevalence and predictors of disability 24-months after injury for hospitalised and non-hospitalised participants: results from a longitudinal cohort study in New Zealand. PLoS One. 2013;8(11):e80194.

30. Harcombe H, Langley J, Davie G, Derrett S. Functional status following injury: what recovery pathways do people follow? Injury. 2015;46(7):1275-80.

31. Langley J, Davie G, Wilson S, et al. Difficulties in functioning 1 year after injury: the role of preinjury sociodemographic and health characteristics, health care and injury-related factors. Arch Phys Med Rehabil. 2013:94(7): $1277-86$.

32. Maclennan B, Wyeth E, Hokowhitu B, Wilson S, Derrett S. Injury severity and 3-month outcomes among Maori: results from a New Zealand prospective cohort study. N Z Med J. 2013;126(1379):39-49.

33. Maclennan B, Wyeth E, Davie G, Wilson S, Derrett S. Twelve-month postinjury outcomes for Maori and non-Maori: findings from a New Zealand cohort study. Aust N Z J Public Health. 2014;38(3):227-33.

34. Mauiliu M, Derrett S, Samaranayaka A, Sopoaga F, Kokaua J, Davie G. Pacific peoples three months after injury: a comparison of outcomes between Pacific and non-Pacific participants in a NZ cohort study. Aust N Z J Public Health. 2013;37(5):463-9.

35. Wilson R, Derrett S, Hansen P, Langley JD. Costs of injury in New Zealand: Accident Compensation Corporation spending, personal spending and quality-adjusted life years lost. Inj Prev. 2013;19(2):124-9.

36. Wyeth EH, Samaranayaka A, Davie G, Derrett S. Prevalence and predictors of disability for Maori 24 months after injury. Aust N Z J Public Health. 2017; 41(3):262-8.

37. Wyeth $E H$, Samaranayaka $A$, Lambert $M$, et al. Understanding longer-term disability outcomes for Māori and non-Māori after hospitalisation for injury: results from a longitudinal cohort study. Public Health. 2018;176:118-27.

38. Dhungel V, Liao J, Raut $\mathrm{H}$, et al. Obesity delays functional recovery in trauma patients. J Surg Res. 2015;193(1):415-20.

39. Dinh MM, Cornwall K, Bein KJ, Gabbe BJ, Tomes BA, Ivers R. Health status and return to work in trauma patients at 3 and 6 months post-discharge: an Australian major trauma Centre study. Eur J Trauma Emerg Surg. 2016;42(4): 483-90.

40. Gabbe BJ, Simpson PM, Sutherland AM, Wolfe R, Lyons RA, Cameron PA. Evaluating time points for measuring recovery after major trauma in adults. Ann Surg. 2013;257(1):166-72.

41. Gabbe BJ, Simpson PM, Harrison JE, et al. Return to work and functiona outcomes after major trauma. Ann Surg. 2016;263(4):623-32. 
42. Gabbe BJ, Simpson PM, Cameron PA, et al. Long-term health status and trajectories of seriously injured patients: a population-based longitudinal study. PLoS Med. 2017;14(7):e1002322.

43. Gross T, Amsler F. Prevalence and incidence of longer term pain in survivors of polytrauma. Surgery. 2011;150(5):985-95.

44. Gross $T$, Schüepp $M$, Attenberger $C$, Pargger $H$, Amsler F. Outcome in polytraumatized patients with and without brain injury. Acta Anaesthesiol Scand. 2012;56(9):1163-74.

45. Gross T, Morell S, Amsler F. Gender-specific improvements in outcome 1 and 2 years after major trauma. J Surg Res. 2019;235:459-69.

46. Innocenti F, Coppa A, Del Taglia B, et al. Prognosis and health-related quality of life in elderly patients after a mild to moderate trauma. Intern Emerg Med. 2014;9(4):467-74

47. Innocenti F, Del Taglia B, Coppa A, et al. Quality of life after mild to moderate trauma. Injury. 2015;46(5):902-8.

48. Jagnoor J, Prinja S, Christou A, Baker J, Gabbe B, Ivers R. Health-related quality of life and function after paediatric injuries in India: a longitudinal study. Int J Environ Res Public Health. 2017;14(10):1144

49. Kendrick D, Kelllezi B, Coupland C, et al. Psychological morbidity and healthrelated quality of life after injury: multicentre cohort study. Qual Life Res. 2017:26(5):1233-50.

50. Llaquet Bayo H, Montmany S, Rebasa P, Secanella M, Alberich M, Navarro S. Analysis of quality of life after major trauma: a spanish follow-up cohort study. Eur J Trauma Emerg Surg. 2019;45(2):289-97.

51. Nguyen $H$, Ivers $R$, Pham C. Health-related quality of life and recovery patterns among hospitalised injury patients in Vietnam. Qual Life Res. 2018; 27(3):619-29.

52. Orwelius L, Bergkvist M, Nordlund A, et al. Physical effects of trauma and the psychological consequences of preexisting diseases account for a significant portion of the health-related quality of life patterns of former trauma patients. J Trauma Acute Care Surg. 2012;72(2):504-12.

53. Pieper P, Garvan C. Concordance of child and parent reports of healthrelated quality of life in children with mild traumatic brain or non-brain injuries and in uninjured children: longitudinal evaluation. J Pediatr Health Care. 2015;29(4):343-51.

54. Rainer $\mathrm{TH}$, Yeung $\mathrm{HH}$, Gabbe BJ, et al. A comparison of functional outcome in patients sustaining major trauma: a multicentre, prospective, international study. PloS One. 2014;9(8):e103396.

55. Rainer TH, Yeung JHH, Cheung SKC, et al. Assessment of quality of life and functional outcome in patients sustaining moderate and major trauma: a multicentre, prospective cohort study. Injury. 2014;45(5):902-9.

56. Ringdal M, Plos K, Ortenwall P, Bergbom I. Memories and health-related quality of life after intensive care: a follow-up study. Crit Care Med. 2010 38(1):38-44.

57. Rivara FP, McCarty CA, Shandro J, Wang J, Zatzick D. Parental injury and psychological health of children. Pediatrics. 2014;134(1):e88-97.

58. Schneeberg A, Ishikawa T, Kruse $\mathrm{S}$, et al. A longitudinal study on quality of life after injury in children. Health Qual Life Outcomes. 2016;14(1):120.

59. Soberg HL, Finset A, Roise O, Bautz-Holter E. The trajectory of physical and mental health from injury to 5 years after multiple trauma: a prospective, longitudinal cohort study. Arch Phys Med Rehabil. 2012;93(5):765-74.

60. Soberg HL, Bautz-Holter E, Finset A, Roise O, Andelic N. Physical and mental health 10 years after multiple trauma: a prospective cohort study. J Trauma Acute Care Surg. 2015;78(3):628-33.

61. Tamura N, Kuriyama A, Kaihara T. Health-related quality of life in trauma patients at 12 months after injury: a prospective cohort study. Eur J Trauma Emerg Surg. 2018;45:1107-13.

62. Tøien K, Bredal IS, Skogstad L, Myhren H, Ekeberg $\varnothing$. Health related quality of life in trauma patients. Data from a one-year follow up study compared with the general population. Scand J Trauma Resusc Emerg Med. 2011; 19(1):22.

63. Yiengprugsawan $V$, Berecki-Gisolf J, McClure R, et al. The effect of injuries on health measured by short form 8 among a large cohort of Thai adults. PLoS One. 2014;9(2):e88903.

64. Zarzaur BL, Bell T. Trajectory subtypes after injury and patient-centered outcomes. J Surg Res. 2016;202(1):103-10.

65. Zarzaur BL, Bell TM, Zanskas SA. Resiliency and quality of life trajectories after injury. J Trauma Acute Care Surg. 2017;82(5):939-45.

66. Neugebauer E, Bouillon B, Bullinger M, Wood-Dauphinée S. Quality of life after multiple trauma-summary and recommendations of the consensus conference. Restor Neurol Neurosci. 2002;20(3-4):161-7.
67. Wilson R, Derrett S, Hansen P, Langley J. Retrospective evaluation versus population norms for the measurement of baseline health status. Health Qual Life Outcomes. 2012;10(1):68.

68. Cameron CM, Purdie DM, Kliewer EV, McClure RJ. Differences in prevalence of pre-existing morbidity between injured and non-injured populations. Bull World Health Organ. 2005;83(5):345-52.

69. Scholten AC, Haagsma JA, Steyerberg EW, van Beeck EF, Polinder S. Assessment of pre-injury health-related quality of life: a systematic review. Popul Health Metrics. 2017;15(1):10.

\section{Publisher's Note}

Springer Nature remains neutral with regard to jurisdictional claims in published maps and institutional affiliations.
Ready to submit your research? Choose BMC and benefit from:

- fast, convenient online submission

- thorough peer review by experienced researchers in your field

- rapid publication on acceptance

- support for research data, including large and complex data types

- gold Open Access which fosters wider collaboration and increased citations

- maximum visibility for your research: over $100 \mathrm{M}$ website views per year

At BMC, research is always in progress.

Learn more biomedcentral.com/submissions 